Supporting Information for

\title{
Extended Barbaralanes - Sigmatropic Shiftamers or D-Polyacenes?
}

\author{
Dean J. Tantillo, ${ }^{\S \ddagger}$ Roald Hoffmann, ${ }^{\S}$ Kendall N. Houk, ${ }^{\#}$ \\ Philip M. Warner, ${ }^{\Omega}$ Eric C. Brown, ${ }^{*}$ and Daven K. Henze ${ }^{¥}$ \\ $\S^{\S}$ Department of Chemistry and Chemical Biology \\ Cornell University \\ Ithaca, New York 14853-1301 \\ \# Department of Chemistry and Biochemistry \\ University of California, \\ Los Angeles, CA 90095-1569 \\ ${ }^{\Omega}$ Department of Chemistry and Chemical Biology \\ Northeastern University \\ Boston, MA 02115 \\ ${ }^{*}$ Department of Chemistry \\ University of Washington \\ Seattle, WA 98195 \\ ‡ current address: \\ Department of Chemistry \\ University of California, Davis \\ One Shields Avenue \\ Davis, CA 95616
}


Coordinates and energies (at the B3LYP/6-31G(d) level unless otherwise noted) for structures in Figure 1 and Tables 2 and 3, along with an expanded discussion of singlet/triplet and HOMO/LUMO gaps.

\section{Coordinates and Energies}

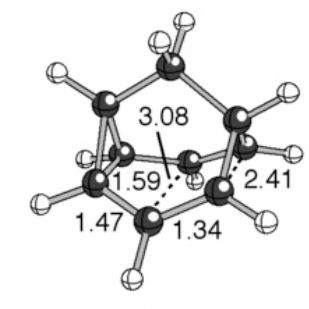

singlet, $C_{\mathrm{s}}$

\begin{tabular}{|c|c|c|c|c|c|}
\hline \multirow{2}{*}{$\begin{array}{l}\text { Center } \\
\text { Number }\end{array}$} & \multirow{2}{*}{$\begin{array}{l}\text { Atomic } \\
\text { Number }\end{array}$} & \multirow{2}{*}{$\begin{array}{c}\text { Atomic } \\
\text { Type }\end{array}$} & \multicolumn{3}{|c|}{ Coordinates (Angstroms) } \\
\hline & & & $\mathrm{x}$ & $\mathrm{Y}$ & $\mathrm{Z}$ \\
\hline 1 & 1 & & 2.250050 & -1.226092 & -0.524466 \\
\hline 2 & 1 & & 2.251972 & 1.222609 & -0.524424 \\
\hline 3 & 6 & & 1.274880 & 0.792730 & -0.320759 \\
\hline 4 & 6 & & 1.273702 & -0.794501 & -0.320906 \\
\hline 5 & 6 & & 0.109477 & 1.541327 & -0.821060 \\
\hline 6 & 6 & & 0.107220 & -1.541139 & -0.821400 \\
\hline 7 & 6 & & 1.121818 & -0.000822 & 0.953228 \\
\hline 8 & 6 & & -0.259606 & 0.000258 & 1.574530 \\
\hline 9 & 6 & & -1.334672 & 0.000914 & 0.455119 \\
\hline 10 & 6 & & -1.137199 & 1.205490 & -0.446576 \\
\hline 11 & 6 & & -1.138890 & -1.204276 & -0.446034 \\
\hline 12 & 1 & & 0.284172 & 2.349619 & -1.528023 \\
\hline 13 & 1 & & 0.280750 & -2.349405 & -1.528678 \\
\hline 14 & 1 & & 1.964910 & -0.001461 & 1.641092 \\
\hline 15 & 1 & & -0.389708 & 0.887970 & 2.206139 \\
\hline 16 & 1 & & -0.390977 & -0.887073 & 2.206412 \\
\hline 17 & 1 & & -2.335162 & 0.001648 & 0.899573 \\
\hline 18 & 1 & & -2.006979 & 1.724251 & -0.842633 \\
\hline 19 & 1 & & -2.009415 & -1.721955 & -0.841841 \\
\hline
\end{tabular}




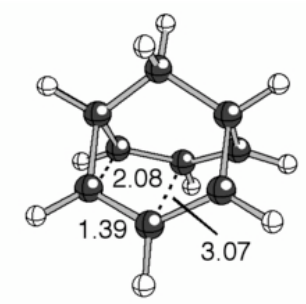

TS, $C_{2 \mathrm{v}}$

\begin{tabular}{|c|c|c|c|c|c|}
\hline \multirow{2}{*}{$\begin{array}{l}\text { Center } \\
\text { Number }\end{array}$} & \multirow{2}{*}{$\begin{array}{l}\text { Atomic } \\
\text { Number }\end{array}$} & \multirow{2}{*}{$\begin{array}{c}\text { Atomic } \\
\text { Type }\end{array}$} & \multicolumn{3}{|c|}{ Coordinates (Angstroms) } \\
\hline & & & $\mathrm{x}$ & $\mathrm{Y}$ & $\mathrm{Z}$ \\
\hline 1 & 1 & & -2.152528 & 1.384994 & -0.783978 \\
\hline 2 & 1 & & -2.152527 & -1.384981 & -0.784001 \\
\hline 3 & 6 & & -1.212582 & -1.041182 & -0.363316 \\
\hline 4 & 6 & & -1.212582 & 1.041189 & -0.363300 \\
\hline 5 & 6 & & 0.000011 & -1.534170 & -0.835614 \\
\hline 6 & 6 & & 0.000009 & 1.534186 & -0.835592 \\
\hline 7 & 6 & & -1.247370 & -0.000005 & 0.712280 \\
\hline 8 & 6 & & -0.000021 & -0.000019 & 1.599331 \\
\hline 9 & 6 & & 1.247352 & -0.000003 & 0.712315 \\
\hline 10 & 6 & & 1.212592 & -1.041155 & -0.363301 \\
\hline 11 & 6 & & 1.212591 & 1.041164 & -0.363285 \\
\hline 12 & 1 & & 0.000025 & -2.235038 & -1.667440 \\
\hline 13 & 1 & & 0.000023 & 2.235065 & -1.667408 \\
\hline 14 & 1 & & -2.172850 & -0.000008 & 1.294113 \\
\hline 15 & 1 & & -0.000029 & -0.887744 & 2.242661 \\
\hline 16 & 1 & & -0.000030 & 0.887672 & 2.242708 \\
\hline 17 & 1 & & 2.172819 & -0.000006 & 1.294168 \\
\hline 18 & 1 & & 2.152549 & -1.384935 & -0.783975 \\
\hline 19 & 1 & & 2.152548 & 1.384950 & -0.783955 \\
\hline
\end{tabular}

$\mathrm{HF}=-348.9022972$

$\mathrm{ZPE}=0.163544$

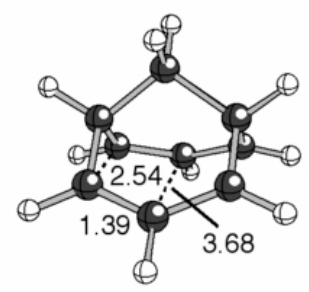

triplet, $C_{2 v}$

\begin{tabular}{|c|c|c|c|c|c|}
\hline \multirow{2}{*}{$\begin{array}{l}\text { Center } \\
\text { Number }\end{array}$} & \multirow{2}{*}{$\begin{array}{l}\text { Atomic } \\
\text { Number }\end{array}$} & \multirow{2}{*}{$\begin{array}{c}\text { Atomic } \\
\text { Type }\end{array}$} & \multicolumn{3}{|c|}{ Coordinates (Angstroms) } \\
\hline & & & $\mathrm{x}$ & $\mathrm{Y}$ & $\mathrm{z}$ \\
\hline 1 & 1 & & -1.714490 & -2.154420 & -0.645849 \\
\hline 2 & 1 & & 1.715368 & -2.153558 & -0.645478 \\
\hline 3 & 6 & & 1.270145 & -1.216122 & -0.323728 \\
\hline 4 & 6 & & -1.269397 & -1.216505 & -0.323967 \\
\hline 5 & 6 & & 1.838342 & 0.000426 & -0.684319 \\
\hline 6 & 6 & & -1.838356 & -0.000186 & -0.684352 \\
\hline 7 & 6 & & 0.000230 & -1.246307 & 0.513316 \\
\hline 8 & 6 & & -0.000087 & -0.000090 & 1.429506 \\
\hline
\end{tabular}




\begin{tabular}{rrrrr}
9 & 6 & -0.000249 & 1.246288 & 0.513352 \\
10 & 6 & 1.269484 & 1.216528 & -0.323792 \\
11 & 6 & -1.270032 & 1.216086 & -0.323708 \\
12 & 1 & 2.752160 & 0.000465 & -1.276487 \\
13 & 1 & -2.752179 & -0.000604 & -1.276492 \\
14 & 1 & 0.000349 & -2.161540 & 1.119992 \\
15 & 1 & 0.888430 & -0.000352 & 2.070542 \\
16 & 1 & -0.888729 & -0.000729 & 2.070386 \\
17 & 1 & -0.000488 & 2.161885 & 1.119435 \\
18 & 1 & 1.714265 & 2.154335 & -0.645083 \\
19 & 1 & -1.715171 & 2.153804 & -0.644813 \\
\hline
\end{tabular}

$\mathrm{HF}=-348.8657195$

$\mathrm{ZPE}=0.160950$

$\mathrm{S} 2=2.060778$

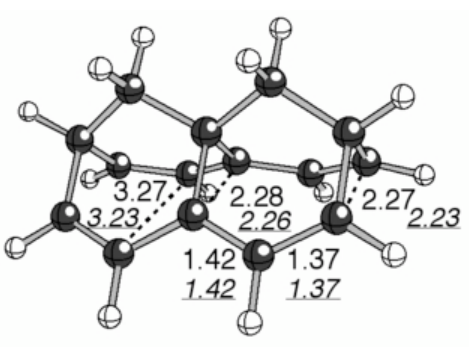

singlet, $C_{2 v}$

B 3 L Y P / $6-31 \mathrm{G} \mathrm{(d)}$

\begin{tabular}{|c|c|c|c|c|c|}
\hline \multirow{2}{*}{$\begin{array}{l}\text { Center } \\
\text { Number }\end{array}$} & \multirow{2}{*}{$\begin{array}{l}\text { Atomic } \\
\text { Number }\end{array}$} & \multirow{2}{*}{$\begin{array}{c}\text { Atomic } \\
\text { Type }\end{array}$} & \multicolumn{3}{|c|}{ Coordinates (Angstroms) } \\
\hline & & & $\mathrm{x}$ & $\mathrm{Y}$ & $\mathrm{Z}$ \\
\hline 1 & 6 & & -2.445852 & 1.135951 & -0.370664 \\
\hline 2 & 6 & & -2.445824 & -1.135935 & -0.370742 \\
\hline 3 & 6 & & -1.242266 & 1.634400 & -0.791739 \\
\hline 4 & 6 & & -1.242230 & -1.634360 & -0.791826 \\
\hline 5 & 6 & & -2.502718 & -0.000026 & 0.615929 \\
\hline 6 & 6 & & -0.000068 & 1.140995 & -0.313313 \\
\hline 7 & 6 & & -0.000041 & -1.140979 & -0.313353 \\
\hline 8 & 6 & & 1.242223 & 1.634694 & -0.791684 \\
\hline 9 & 6 & & 1.242258 & -1.634688 & -0.791697 \\
\hline 10 & 6 & & 0.000040 & -0.000010 & 0.690918 \\
\hline 11 & 6 & & 2.445785 & -1.136407 & -0.370629 \\
\hline 12 & 6 & & 2.445770 & 1.136380 & -0.370691 \\
\hline 13 & 1 & & 3.374097 & -1.494659 & -0.806578 \\
\hline 14 & 1 & & 3.374605 & 1.494860 & -0.806894 \\
\hline 15 & 6 & & 2.502730 & 0.000007 & 0.615463 \\
\hline 16 & 6 & & -1.278227 & -0.000040 & 1.538647 \\
\hline 17 & 6 & & 1.278393 & -0.000009 & 1.538442 \\
\hline 18 & 1 & & 1.220052 & 2.411047 & -1.555213 \\
\hline 19 & 1 & & 1.220105 & -2.411020 & -1.555247 \\
\hline 20 & 1 & & -3.374441 & 1.494028 & -0.806998 \\
\hline 21 & 1 & & -3.374404 & -1.494002 & -0.807104 \\
\hline 22 & 1 & & -1.220332 & 2.410503 & -1.555515 \\
\hline 23 & 1 & & -1.220281 & -2.410429 & -1.555637 \\
\hline 24 & 1 & & -3.435688 & -0.000054 & 1.189079 \\
\hline
\end{tabular}




\begin{tabular}{|c|c|c|c|c|c|}
\hline 25 & 1 & & 3.435716 & 0.000018 & 1.188701 \\
\hline 26 & 1 & & -1.293416 & 0.887029 & 2.183371 \\
\hline 27 & 1 & & -1.293405 & -0.887142 & 2.183327 \\
\hline 28 & 1 & & 1.293773 & 0.887054 & 2.183175 \\
\hline 29 & 1 & & 1.293778 & -0.887076 & 2.183170 \\
\hline \multicolumn{6}{|c|}{$\begin{array}{l}\mathrm{HF}=-579.9561105 \\
\mathrm{ZPE}=0.246725\end{array}$} \\
\hline \multicolumn{6}{|c|}{ MP $2 / 6-31 G(d)$} \\
\hline Center & Atomic & Atomic & \multicolumn{3}{|c|}{ Coordinates (Angstroms) } \\
\hline Number & Number & Type & $\mathrm{x}$ & $\mathrm{Y}$ & $\mathrm{z}$ \\
\hline 1 & 6 & & -2.444926 & 1.114575 & -0.367194 \\
\hline 2 & 6 & & -2.444695 & -1.114681 & -0.367456 \\
\hline 3 & 6 & & -1.239810 & 1.614865 & -0.797055 \\
\hline 4 & 6 & & -1.239502 & -1.614883 & -0.797232 \\
\hline 5 & 6 & & -2.492478 & -0.000159 & 0.629992 \\
\hline 6 & 6 & & -0.000113 & 1.129681 & -0.313641 \\
\hline 7 & 6 & & 0.000110 & -1.129681 & -0.313651 \\
\hline 8 & 6 & & 1.239497 & 1.614893 & -0.797223 \\
\hline 9 & 6 & & 1.239811 & -1.614858 & -0.797064 \\
\hline 10 & 6 & & 0.000005 & 0.000003 & 0.685210 \\
\hline 11 & 6 & & 2.444929 & -1.114585 & -0.367191 \\
\hline 12 & 6 & & 2.444691 & 1.114683 & -0.367453 \\
\hline 13 & 1 & & 3.377918 & -1.471929 & -0.799590 \\
\hline 14 & 1 & & 3.377604 & 1.472140 & -0.799930 \\
\hline 15 & 6 & & 2.492483 & 0.000163 & 0.629985 \\
\hline 16 & 6 & & -1.265662 & -0.000118 & 1.533666 \\
\hline 17 & 6 & & 1.265659 & 0.000104 & 1.533663 \\
\hline 18 & 1 & & 1.223076 & 2.379872 & -1.574553 \\
\hline 19 & 1 & & 1.223525 & -2.379856 & -1.574379 \\
\hline 20 & 1 & & -3.377920 & 1.471926 & -0.799579 \\
\hline 21 & 1 & & -3.377617 & -1.472118 & -0.799927 \\
\hline 22 & 1 & & -1.223517 & 2.379860 & -1.574372 \\
\hline 23 & 1 & & -1.223076 & -2.379854 & -1.574570 \\
\hline 24 & 1 & & -3.421396 & -0.000288 & 1.210843 \\
\hline 25 & 1 & & 3.421394 & 0.000275 & 1.210847 \\
\hline 26 & 1 & & -1.271154 & 0.888969 & 2.177807 \\
\hline 27 & 1 & & -1.271039 & -0.889263 & 2.177724 \\
\hline 28 & 1 & & 1.271048 & 0.889243 & 2.177735 \\
\hline 29 & 1 & & 1.271156 & -0.888983 & 2.177801 \\
\hline
\end{tabular}

CASSCF $(10,10) / 6-31 G *$ optimization

$\begin{array}{llll}6 & 0.0000000 & 0.0000000 & 0.6709756 \\ 6 & -1.1533947 & 0.0000000 & -0.3104736 \\ 6 & -1.6760366 & 1.2475036 & -0.7674181 \\ 6 & 0.0000000 & 2.4950443 & 0.5928637 \\ 6 & 1.1835578 & 2.4473495 & -0.3414992 \\ 6 & 0.0000000 & 1.2743488 & 1.5156589 \\ 1 & -2.4727236 & 1.2264741 & -1.4923539 \\ 1 & 0.0000000 & 3.4107749 & 1.1740274 \\ 1 & -1.5636023 & 3.3680703 & -0.7474682\end{array}$




$\begin{array}{llll}1 & 0.8780638 & 1.2907533 & 2.1546722 \\ 6 & 1.1533947 & 0.0000000 & -0.3104736 \\ 6 & 1.6760366 & 1.2475036 & -0.7674181 \\ 6 & -1.1835578 & 2.4473495 & -0.3414992 \\ 1 & 2.4727236 & 1.2264741 & -1.4923539 \\ 1 & 1.5636023 & 3.3680703 & -0.7474682 \\ 1 & -0.8780638 & 1.2907533 & 2.1546722 \\ 6 & 1.1533947 & 0.0000000 & -0.3104736 \\ 6 & 1.6760366 & -1.2475036 & -0.7674181 \\ 6 & 0.0000000 & -2.4950443 & 0.5928637 \\ 6 & -1.1835578 & -2.4473495 & -0.3414992 \\ 6 & 0.000000 & -1.2743488 & 1.5156589 \\ 1 & 2.4727236 & -1.2264741 & -1.4923539 \\ 1 & 0.0000000 & -3.4107749 & 1.1740274 \\ 1 & 1.5636023 & -3.3680703 & -0.7474682 \\ 1 & -0.8780638 & -1.2907533 & 2.1546722 \\ 6 & -1.1533947 & 0.0000000 & -0.3104736 \\ 6 & -1.6760366 & -1.2475036 & -0.7674181 \\ 6 & 1.1835578 & -2.4473495 & -0.3414992 \\ 1 & -2.4727236 & -1.2264741 & -1.4923539 \\ 1 & -1.5636023 & -3.3680703 & -0.7474682 \\ 1 & 0.8780638 & -1.2907533 & 2.1546722\end{array}$

$\operatorname{CASSCF}(10,10) / 6-31 \mathrm{G} *$ Energy $=-576.2245765$

CASPT2//CASSCF Energy $=-578.1130103$

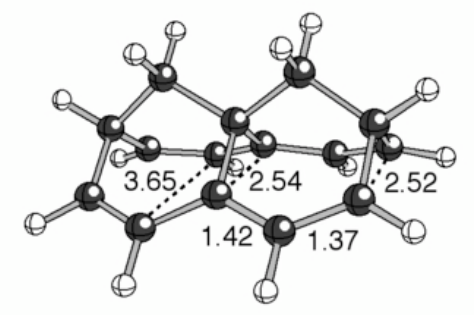

triplet, $C_{2 v}$

\begin{tabular}{|c|c|c|c|c|c|}
\hline \multirow{2}{*}{$\begin{array}{l}\text { Center } \\
\text { Number }\end{array}$} & \multirow{2}{*}{$\begin{array}{l}\text { Atomic } \\
\text { Number }\end{array}$} & \multirow{2}{*}{$\begin{array}{c}\text { Atomic } \\
\text { Type }\end{array}$} & \multicolumn{3}{|c|}{ Coordinates (Angstroms) } \\
\hline & & & $\mathrm{x}$ & $\mathrm{Y}$ & $\mathrm{z}$ \\
\hline 1 & 6 & & -2.446925 & 1.262276 & -0.359793 \\
\hline 2 & 6 & & -2.446893 & -1.262293 & -0.359832 \\
\hline 3 & 6 & & -1.244634 & 1.825418 & -0.691418 \\
\hline 4 & 6 & & -1.244593 & -1.825419 & -0.691449 \\
\hline 5 & 6 & & -2.499490 & -0.000022 & 0.485935 \\
\hline 6 & 6 & & -0.000005 & 1.267592 & -0.298175 \\
\hline 7 & 6 & & 0.000028 & -1.267592 & -0.298181 \\
\hline 8 & 6 & & 1.244404 & 1.825455 & -0.691374 \\
\hline 9 & 6 & & 1.244445 & -1.825449 & -0.691348 \\
\hline 10 & 6 & & 0.000046 & 0.000003 & 0.571951 \\
\hline 11 & 6 & & 2.446878 & -1.262398 & -0.359870 \\
\hline 12 & 6 & & 2.446843 & 1.262423 & -0.359895 \\
\hline 13 & 1 & & 3.377148 & -1.697905 & -0.713754 \\
\hline 14 & 1 & & 3.377128 & 1.697932 & -0.713833 \\
\hline 15 & 6 & & 2.499726 & 0.000023 & 0.485598 \\
\hline 16 & 6 & & -1.280073 & -0.000024 & 1.430647 \\
\hline 17 & 6 & & 1.280297 & 0.000011 & 1.430452 \\
\hline 18 & 1 & & 1.225604 & 2.729962 & -1.298771 \\
\hline 19 & 1 & & 1.225657 & -2.729972 & -1.298724 \\
\hline
\end{tabular}




\begin{tabular}{rrrrr}
20 & 1 & -3.377687 & 1.697509 & -0.713984 \\
21 & 1 & -3.377645 & -1.697515 & -0.714063 \\
22 & 1 & -1.225830 & 2.729796 & -1.298991 \\
23 & 1 & -1.225776 & -2.729782 & -1.299046 \\
24 & 1 & -3.430098 & -0.000046 & 1.068328 \\
25 & 1 & 3.430503 & 0.000037 & 1.067882 \\
26 & 1 & -1.298003 & 0.887660 & 2.074013 \\
27 & 1 & -1.297983 & -0.887726 & 2.073986 \\
28 & 1 & 1.298324 & 0.887735 & 2.073727 \\
29 & 1 & 1.298336 & -0.887708 & 2.073733 \\
\hline
\end{tabular}

$\mathrm{HF}=-579.923802$

$\mathrm{ZPE}=0.244257$

$\mathrm{S} 2=2.090586$

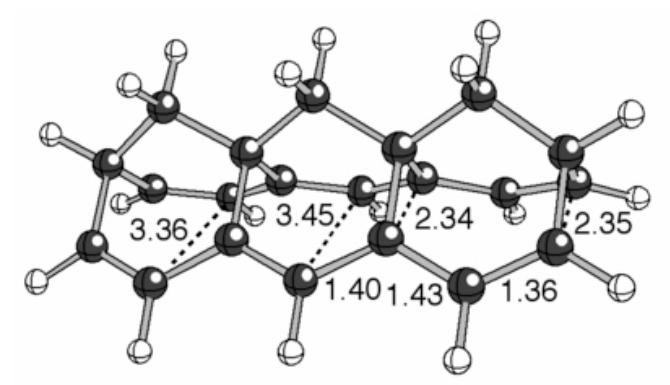

singlet, $C_{2 \mathrm{v}}$

\begin{tabular}{|c|c|c|c|c|c|}
\hline \multirow{2}{*}{$\begin{array}{l}\text { Center } \\
\text { Number }\end{array}$} & \multirow{2}{*}{$\begin{array}{l}\text { Atomic } \\
\text { Number }\end{array}$} & \multirow{2}{*}{$\begin{array}{l}\text { Atomic } \\
\text { Type }\end{array}$} & \multicolumn{3}{|c|}{ Coordinates (Angstroms) } \\
\hline & & & $\mathrm{x}$ & $\mathrm{Y}$ & $\mathrm{Z}$ \\
\hline 1 & 6 & & 0.000000 & 0.000000 & 0.000000 \\
\hline 2 & 6 & & 0.000000 & 0.000000 & 2.348000 \\
\hline 3 & 6 & & 1.262051 & 0.000000 & -0.507853 \\
\hline 4 & 6 & & 1.261870 & 0.000661 & 2.856036 \\
\hline 5 & 6 & & -0.357345 & 0.881358 & 1.174024 \\
\hline 6 & 6 & & 2.301212 & 0.842974 & 0.002208 \\
\hline 7 & 6 & & 2.300751 & 0.844238 & 2.346121 \\
\hline 8 & 6 & & 3.602650 & 0.804054 & -0.500785 \\
\hline 9 & 6 & & 3.602171 & 0.805855 & 2.849202 \\
\hline 10 & 6 & & 1.984396 & 1.762369 & 1.174070 \\
\hline 11 & 6 & & 4.647448 & 1.582270 & 2.346378 \\
\hline 12 & 6 & & 4.648325 & 1.579979 & 0.002248 \\
\hline 13 & 6 & & 5.982064 & 1.485154 & 2.856554 \\
\hline 14 & 6 & & 5.983142 & 1.481346 & -0.507671 \\
\hline 15 & 6 & & 4.381621 & 2.517577 & 1.174432 \\
\hline 16 & 6 & & 0.499746 & 2.154425 & 1.174103 \\
\hline 17 & 6 & & 2.920688 & 2.976604 & 1.173969 \\
\hline 18 & 1 & & 3.821095 & 0.108537 & -1.310847 \\
\hline 19 & 1 & & 3.820858 & 0.110399 & 3.659118 \\
\hline 20 & 6 & & 5.375102 & 3.688632 & 1.174430 \\
\hline 21 & 6 & & 6.806229 & 3.134395 & 1.174504 \\
\hline 22 & 6 & & 7.017336 & 2.206969 & 2.348733 \\
\hline 23 & 6 & & 7.018308 & 2.203737 & 0.001422 \\
\hline 24 & 1 & & -0.745626 & -0.696281 & -0.374949 \\
\hline 25 & 1 & & -0.745626 & -0.696281 & 2.722949 \\
\hline 26 & 1 & & 1.519391 & -0.682195 & -1.317822 \\
\hline 27 & 1 & & 1.519483 & -0.681533 & 3.664978 \\
\hline
\end{tabular}




\begin{tabular}{|c|c|c|c|c|}
\hline 28 & 1 & -1.425890 & 1.126478 & 1.174057 \\
\hline 29 & 1 & 6.161435 & 0.777423 & 3.665498 \\
\hline 30 & 1 & 6.162226 & 0.774448 & -1.316598 \\
\hline 31 & 1 & 0.277312 & 2.759665 & 0.286996 \\
\hline 32 & 1 & 0.277357 & 2.759557 & 2.062036 \\
\hline 33 & 1 & 2.726951 & 3.593838 & 0.287901 \\
\hline 34 & 1 & 2.726781 & 3.594002 & 2.060876 \\
\hline 35 & 1 & 5.211460 & 4.312180 & 2.061403 \\
\hline 36 & 1 & 5.211379 & 4.312334 & 0.287333 \\
\hline 37 & 1 & 7.541607 & 3.946256 & 1.173969 \\
\hline 38 & 1 & 8.026849 & 2.063165 & 2.723815 \\
\hline 39 & 1 & 8.028187 & 2.058480 & -0.374433 \\
\hline
\end{tabular}

$\mathrm{HF}=-811.0083968$

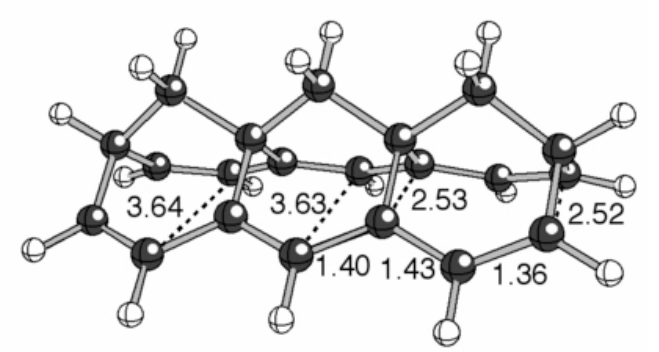

triplet, $C_{2 v}$

\begin{tabular}{|c|c|c|c|c|c|}
\hline \multirow{2}{*}{$\begin{array}{l}\text { Center } \\
\text { Number }\end{array}$} & \multirow{2}{*}{$\begin{array}{l}\text { Atomic } \\
\text { Number }\end{array}$} & \multirow{2}{*}{$\begin{array}{c}\text { Atomic } \\
\text { Type }\end{array}$} & \multicolumn{3}{|c|}{ Coordinates (Angstroms) } \\
\hline & & & $\mathrm{x}$ & $\mathrm{Y}$ & $\mathrm{Z}$ \\
\hline 1 & 6 & & -3.678228 & 1.258205 & -0.410263 \\
\hline 2 & 6 & & -3.678062 & -1.257793 & -0.412768 \\
\hline 3 & 6 & & -2.477344 & 1.820575 & -0.714081 \\
\hline 4 & 6 & & -2.476943 & -1.819398 & -0.717075 \\
\hline 5 & 6 & & -3.750828 & -0.000701 & 0.438432 \\
\hline 6 & 6 & & -1.230185 & 1.264336 & -0.284013 \\
\hline 7 & 6 & & -1.230084 & -1.263851 & -0.285248 \\
\hline 8 & 6 & & -0.000367 & 1.817878 & -0.642953 \\
\hline 9 & 6 & & -0.000004 & -1.816515 & -0.644645 \\
\hline 10 & 6 & & -1.254726 & -0.000442 & 0.587916 \\
\hline 11 & 6 & & 1.230040 & -1.263969 & -0.284947 \\
\hline 12 & 6 & & 1.229939 & 1.264780 & -0.285000 \\
\hline 13 & 6 & & 2.476952 & -1.819635 & -0.716470 \\
\hline 14 & 6 & & 2.476551 & 1.821470 & -0.716068 \\
\hline 15 & 6 & & 1.255004 & -0.000878 & 0.589412 \\
\hline 16 & 6 & & -2.556457 & -0.001167 & 1.413392 \\
\hline 17 & 6 & & 0.000593 & -0.001213 & 1.477386 \\
\hline 18 & 1 & & -2.441569 & 2.722186 & -1.325212 \\
\hline 19 & 1 & & -2.440728 & -2.719786 & -1.329982 \\
\hline 20 & 1 & & -4.695646 & -0.001367 & 0.997826 \\
\hline 21 & 1 & & -0.000774 & 2.717500 & -1.259112 \\
\hline 22 & 1 & & 0.000031 & -2.714909 & -1.262594 \\
\hline 23 & 1 & & 2.440800 & -2.719966 & -1.329463 \\
\hline 24 & 1 & & 2.439959 & 2.723016 & -1.327249 \\
\hline 25 & 1 & & -2.590238 & 0.886375 & 2.057067 \\
\hline 26 & 1 & & -2.590182 & -0.889778 & 2.055594 \\
\hline 27 & 1 & & 0.000866 & 0.885246 & 2.124114 \\
\hline
\end{tabular}




\begin{tabular}{rrrrr}
28 & 1 & 0.000884 & -0.888811 & 2.122550 \\
29 & 6 & 2.557033 & -0.001857 & 1.414417 \\
30 & 1 & 2.590970 & -0.890642 & 2.056366 \\
31 & 1 & 2.591066 & 0.885510 & 2.058321 \\
32 & 6 & 3.751093 & -0.000721 & 0.439512 \\
33 & 1 & 4.696094 & -0.000845 & 0.998598 \\
34 & 6 & 3.678049 & -1.258145 & -0.411869 \\
35 & 6 & 3.677882 & 1.259535 & -0.413215 \\
36 & 1 & -4.601320 & 1.688575 & -0.789498 \\
37 & 1 & -4.601096 & -1.687529 & -0.792858 \\
38 & 1 & 4.600504 & 1.690184 & -0.793273 \\
39 & 1 & 4.600928 & -1.687915 & -0.792300 \\
\hline
\end{tabular}

$\mathrm{HF}=-810.9760519$

$\mathrm{ZPE}=0.326864$

$\mathrm{S} 2=2.117688$

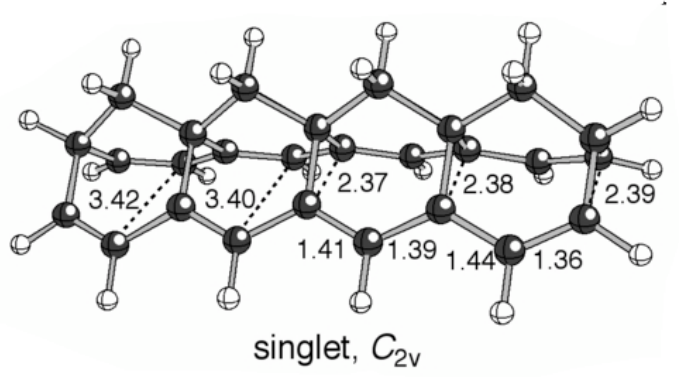

\begin{tabular}{rrrrr} 
Center & $\begin{array}{c}\text { Atomic } \\
\text { Number }\end{array}$ & $\begin{array}{c}\text { Atomic } \\
\text { Number }\end{array}$ & \multicolumn{3}{c}{ Coordinates } & \multicolumn{1}{c}{ (Angstroms) } \\
-1 & 6 & -4.907968 & 1.192877 & -0.473213 \\
2 & 6 & -4.908233 & -1.192813 & -0.472675 \\
3 & 6 & -3.700735 & 1.710735 & -0.812026 \\
4 & 6 & -3.700977 & -1.710817 & -0.811421 \\
5 & 6 & -5.006042 & 0.000206 & 0.452822 \\
6 & 6 & -2.461562 & 1.191553 & -0.299453 \\
7 & 6 & -2.461839 & -1.191641 & -0.298981 \\
8 & 6 & -1.233138 & 1.698754 & -0.689198 \\
9 & 6 & -1.233403 & -1.698844 & -0.688709 \\
10 & 6 & -2.511701 & 0.000072 & 0.653414 \\
11 & 6 & -0.000039 & -1.184817 & -0.241840 \\
12 & 6 & -3.830344 & 0.000325 & 1.442243 \\
13 & 6 & -1.277997 & -0.000020 & 1.564750 \\
14 & 1 & -1.214023 & 2.524425 & -1.400577 \\
15 & 1 & -1.214517 & -2.524808 & -1.399860 \\
16 & 6 & 4.908076 & 1.193522 & -0.472560 \\
17 & 6 & 4.908068 & -1.193220 & -0.473034 \\
18 & 6 & 3.700881 & 1.711419 & -0.811188 \\
19 & 6 & 3.700992 & -1.711128 & -0.811861 \\
20 & 6 & 5.006072 & 0.000008 & 0.452411 \\
21 & 6 & 2.461580 & 1.191765 & -0.298932 \\
22 & 6 & 2.461571 & -1.191677 & -0.299604 \\
23 & 6 & 1.233276 & 1.698703 & -0.688769 \\
24 & 6 & 1.233303 & -1.698849 & -0.689197 \\
25 & 6 & 2.511740 & -0.000083 & 0.653165 \\
26 & 6 & -0.000167 & 1.184589 & -0.241443 \\
27 & 6 & -0.000034 & -0.000135 & 0.716590
\end{tabular}




\begin{tabular}{|c|c|c|c|c|}
\hline 28 & 6 & 3.830451 & -0.000274 & 1.441969 \\
\hline 29 & 6 & 1.278147 & -0.000214 & 1.564725 \\
\hline 30 & 1 & -5.819230 & 1.577710 & -0.924271 \\
\hline 31 & 1 & -5.819494 & -1.577816 & -0.923574 \\
\hline 32 & 1 & -3.643886 & 2.538110 & -1.517933 \\
\hline 33 & 1 & -3.644392 & -2.538355 & -1.517150 \\
\hline 34 & 1 & -5.963279 & 0.000474 & 0.986598 \\
\hline 35 & 1 & -3.877564 & 0.887626 & 2.084869 \\
\hline 36 & 1 & -3.877660 & -0.886705 & 2.085244 \\
\hline 37 & 1 & -1.294032 & 0.886505 & 2.211213 \\
\hline 38 & 1 & -1.294192 & -0.886538 & 2.211249 \\
\hline 39 & 1 & 5.819308 & 1.578623 & -0.923458 \\
\hline 40 & 1 & 5.819437 & -1.578068 & -0.923887 \\
\hline 41 & 1 & 3.644054 & 2.539682 & -1.516045 \\
\hline 42 & 1 & 3.644081 & -2.539049 & -1.517128 \\
\hline 43 & 1 & 5.963415 & -0.000214 & 0.986049 \\
\hline 44 & 1 & 1.214257 & 2.524311 & -1.400284 \\
\hline 45 & 1 & 1.214066 & -2.524412 & -1.400772 \\
\hline 46 & 1 & 3.877638 & 0.886744 & 2.084973 \\
\hline 47 & 1 & 3.877667 & -0.887599 & 2.084528 \\
\hline 48 & 1 & 1.294352 & 0.886240 & 2.211288 \\
\hline 49 & 1 & 1.294130 & -0.886852 & 2.211011 \\
\hline
\end{tabular}

$\mathrm{HF}=-1042.0592854$

$\mathrm{ZPE}=0.412203$

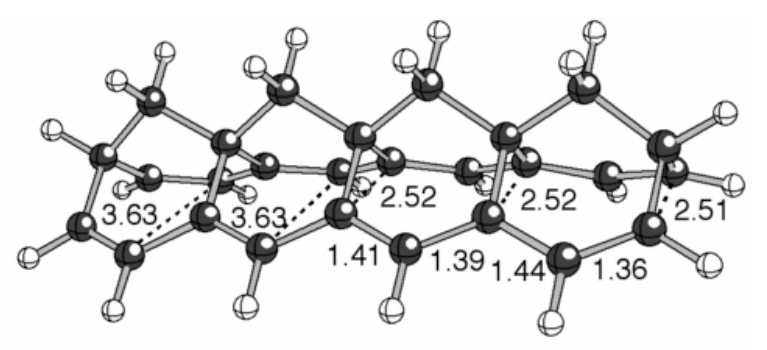

triplet, $C_{2 v}$

\begin{tabular}{|c|c|c|c|c|c|}
\hline \multirow{2}{*}{$\begin{array}{l}\text { Center } \\
\text { Number }\end{array}$} & \multirow{2}{*}{$\begin{array}{l}\text { Atomic } \\
\text { Number }\end{array}$} & \multirow{2}{*}{$\begin{array}{c}\text { Atomic } \\
\text { Type }\end{array}$} & \multicolumn{3}{|c|}{ Coordinates (Angstroms) } \\
\hline & & & $\mathrm{x}$ & $\mathrm{Y}$ & $\mathrm{z}$ \\
\hline 1 & 6 & & -4.907326 & 1.255387 & -0.481384 \\
\hline 2 & 6 & & -4.907623 & -1.255439 & -0.480637 \\
\hline 3 & 6 & & -3.703897 & 1.816464 & -0.758041 \\
\hline 4 & 6 & & -3.704281 & -1.816719 & -0.757172 \\
\hline 5 & 6 & & -5.001779 & 0.000243 & 0.370955 \\
\hline 6 & 6 & & -2.461619 & 1.262076 & -0.292485 \\
\hline 7 & 6 & & -2.461867 & -1.262145 & -0.292100 \\
\hline 8 & 6 & & -1.234574 & 1.813671 & -0.620945 \\
\hline 9 & 6 & & -1.234881 & -1.813776 & -0.620704 \\
\hline 10 & 6 & & -2.509501 & 0.000121 & 0.582607 \\
\hline 11 & 6 & & -0.000119 & -1.261594 & -0.226607 \\
\hline 12 & 6 & & -3.831930 & 0.000392 & 1.374577 \\
\hline 13 & 6 & & -1.278192 & 0.000157 & 1.504396 \\
\hline 14 & 1 & & -1.217595 & 2.710715 & -1.240559 \\
\hline 15 & 1 & & -1.217913 & -2.710910 & -1.240169 \\
\hline 16 & 6 & & 4.907625 & 1.255353 & -0.480692 \\
\hline
\end{tabular}




\begin{tabular}{|c|c|c|c|c|}
\hline 17 & 6 & 4.907338 & -1.255360 & -0.481399 \\
\hline 18 & 6 & 3.704304 & 1.816661 & -0.757207 \\
\hline 19 & 6 & 3.703915 & -1.816420 & -0.758057 \\
\hline 20 & 6 & 5.001752 & -0.000261 & 0.371014 \\
\hline 21 & 6 & 2.461865 & 1.262144 & -0.292065 \\
\hline 22 & 6 & 2.461611 & -1.262073 & -0.292430 \\
\hline 23 & 6 & 1.234923 & 1.813839 & -0.620612 \\
\hline 24 & 6 & 1.234597 & -1.813681 & -0.620919 \\
\hline 25 & 6 & 2.509481 & -0.000128 & 0.582679 \\
\hline 26 & 6 & 0.000115 & 1.261641 & -0.226607 \\
\hline 27 & 6 & -0.000006 & 0.000007 & 0.647924 \\
\hline 28 & 6 & 3.831907 & -0.000407 & 1.374597 \\
\hline 29 & 6 & 1.278165 & -0.000146 & 1.504435 \\
\hline 30 & 1 & -5.821513 & 1.681748 & -0.886880 \\
\hline 31 & 1 & -5.821916 & -1.682070 & -0.885602 \\
\hline 32 & 1 & -3.653072 & 2.713936 & -1.373293 \\
\hline 33 & 1 & -3.653518 & -2.714540 & -1.371921 \\
\hline 34 & 1 & -5.959855 & 0.000538 & 0.906296 \\
\hline 35 & 1 & -3.881968 & 0.888200 & 2.015979 \\
\hline 36 & 1 & -3.882120 & -0.887095 & 2.016414 \\
\hline 37 & 1 & -1.294782 & 0.887040 & 2.150146 \\
\hline 38 & 1 & -1.294830 & -0.886620 & 2.150277 \\
\hline 39 & 1 & 5.821915 & 1.681879 & -0.885774 \\
\hline 40 & 1 & 5.821520 & -1.681654 & -0.886972 \\
\hline 41 & 1 & 3.653529 & 2.714398 & -1.372080 \\
\hline 42 & 1 & 3.653055 & -2.713814 & -1.373419 \\
\hline 43 & 1 & 5.959802 & -0.000538 & 0.906402 \\
\hline 44 & 1 & 1.217964 & 2.711008 & -1.240033 \\
\hline 45 & 1 & 1.217629 & -2.710716 & -1.240531 \\
\hline 46 & 1 & 3.882131 & 0.887074 & 2.016443 \\
\hline 47 & 1 & 3.881976 & -0.888207 & 2.016019 \\
\hline 48 & 1 & 1.294827 & 0.886618 & 2.150346 \\
\hline 49 & 1 & 1.294724 & -0.887031 & 2.150182 \\
\hline
\end{tabular}

$\mathrm{HF}=-1042.0257735$

$\mathrm{ZPE}=0.409287$

$\mathrm{S} 2=2.144903$

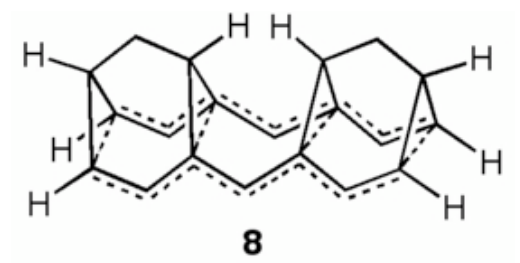

singlet :

\begin{tabular}{|c|c|c|c|c|c|}
\hline \multirow{2}{*}{$\begin{array}{l}\text { Center } \\
\text { Number }\end{array}$} & \multirow{2}{*}{$\begin{array}{l}\text { Atomic } \\
\text { Number }\end{array}$} & \multirow{2}{*}{$\begin{array}{c}\text { Atomic } \\
\text { Type }\end{array}$} & \multicolumn{3}{|c|}{ Coordinates (Angstroms) } \\
\hline & & & $\mathrm{x}$ & $\mathrm{Y}$ & Z \\
\hline 1 & 6 & & 3.671238 & -1.185998 & -0.575211 \\
\hline 2 & 6 & & 3.671240 & 1.186003 & -0.575207 \\
\hline 3 & 6 & & 2.415734 & -1.697452 & -0.689999 \\
\hline 4 & 6 & & 2.415735 & 1.697453 & -0.689998 \\
\hline 5 & 6 & & 3.950383 & 0.000001 & 0.318153 \\
\hline 6 & 6 & & 1.275268 & -1.171840 & 0.012234 \\
\hline 7 & 6 & & 1.275268 & 1.171837 & 0.012230 \\
\hline 8 & 6 & & -0.000001 & -1.641027 & -0.328398 \\
\hline
\end{tabular}




\begin{tabular}{|c|c|c|c|c|}
\hline 9 & 6 & -0.000001 & 1.641022 & -0.328402 \\
\hline 10 & 6 & 1.535650 & 0.000000 & 0.946342 \\
\hline 11 & 6 & -1.275265 & 1.171840 & 0.012232 \\
\hline 12 & 6 & -1.275265 & -1.171845 & 0.012235 \\
\hline 13 & 6 & -2.415737 & 1.697467 & -0.689989 \\
\hline 14 & 6 & -2.415735 & -1.697464 & -0.689993 \\
\hline 15 & 6 & -1.535651 & -0.000002 & 0.946340 \\
\hline 16 & 6 & 2.970299 & 0.000000 & 1.492522 \\
\hline 17 & 1 & 2.235582 & -2.511958 & -1.390009 \\
\hline 18 & 1 & 2.235583 & 2.511957 & -1.390009 \\
\hline 19 & 1 & 4.990237 & 0.000001 & 0.663558 \\
\hline 20 & 1 & 0.000001 & -2.443080 & -1.068239 \\
\hline 21 & 1 & 0.000000 & 2.443077 & -1.068240 \\
\hline 22 & 1 & -2.235583 & 2.511984 & -1.389985 \\
\hline 23 & 1 & -2.235581 & -2.511979 & -1.389992 \\
\hline 24 & 1 & 3.124462 & -0.887048 & 2.117454 \\
\hline 25 & 1 & 3.124460 & 0.887050 & 2.117454 \\
\hline 26 & 6 & -2.970301 & -0.000002 & 1.492515 \\
\hline 27 & 1 & -3.124465 & 0.887046 & 2.117447 \\
\hline 28 & 1 & -3.124467 & -0.887052 & 2.117444 \\
\hline 29 & 6 & -3.950381 & 0.000000 & 0.318140 \\
\hline 30 & 1 & -4.990236 & -0.000002 & 0.663543 \\
\hline 31 & 6 & -3.671240 & 1.186017 & -0.575203 \\
\hline 32 & 6 & -3.671237 & -1.186011 & -0.575210 \\
\hline 33 & 1 & 4.477315 & -1.574133 & -1.192799 \\
\hline 34 & 1 & 4.477317 & 1.574137 & -1.192794 \\
\hline 35 & 1 & -4.477315 & -1.574157 & -1.192790 \\
\hline 36 & 1 & -4.477319 & 1.574167 & -1.192778 \\
\hline 37 & 1 & -0.864685 & 0.000000 & 1.799367 \\
\hline 38 & 1 & 0.864679 & 0.000000 & 1.799365 \\
\hline
\end{tabular}

$\mathrm{HF}=-772.8733192$

$\mathrm{ZPE}=0.323667$

(single point at B3LYP/6-311+G**, $E=-773.06163$ )

triplet:

$\begin{array}{lrrr}6 & 3.660247 & -1.269573 & -0.586374 \\ 6 & 3.660247 & 1.269574 & -0.586372 \\ 6 & 2.423502 & -1.837384 & -0.612555 \\ 6 & 2.423502 & 1.837385 & -0.612553 \\ 6 & 3.923888 & 0.000000 & 0.208807 \\ 6 & 1.272441 & -1.260395 & 0.027727 \\ 6 & 1.272441 & 1.260394 & 0.027729 \\ 6 & 0.000000 & -1.770705 & -0.257311 \\ 6 & 0.000000 & 1.770705 & -0.257308 \\ 6 & 1.523154 & -0.000001 & 0.860750 \\ 6 & -1.272441 & 1.260394 & 0.027729 \\ 6 & -1.272441 & -1.260394 & 0.027727 \\ 6 & -2.423502 & 1.837385 & -0.612553 \\ 6 & -2.423502 & -1.837384 & -0.612555 \\ 6 & -1.523154 & -0.000001 & 0.860751 \\ 6 & 2.963450 & -0.000001 & 1.405250 \\ 1 & 2.268314 & -2.744654 & -1.195203 \\ 1 & 2.268314 & 2.744656 & -1.195199 \\ 1 & 4.968330 & -0.000001 & 0.546358 \\ 1 & 0.000000 & -2.658571 & -0.892622 \\ 1 & 0.000000 & 2.658572 & -0.892618\end{array}$




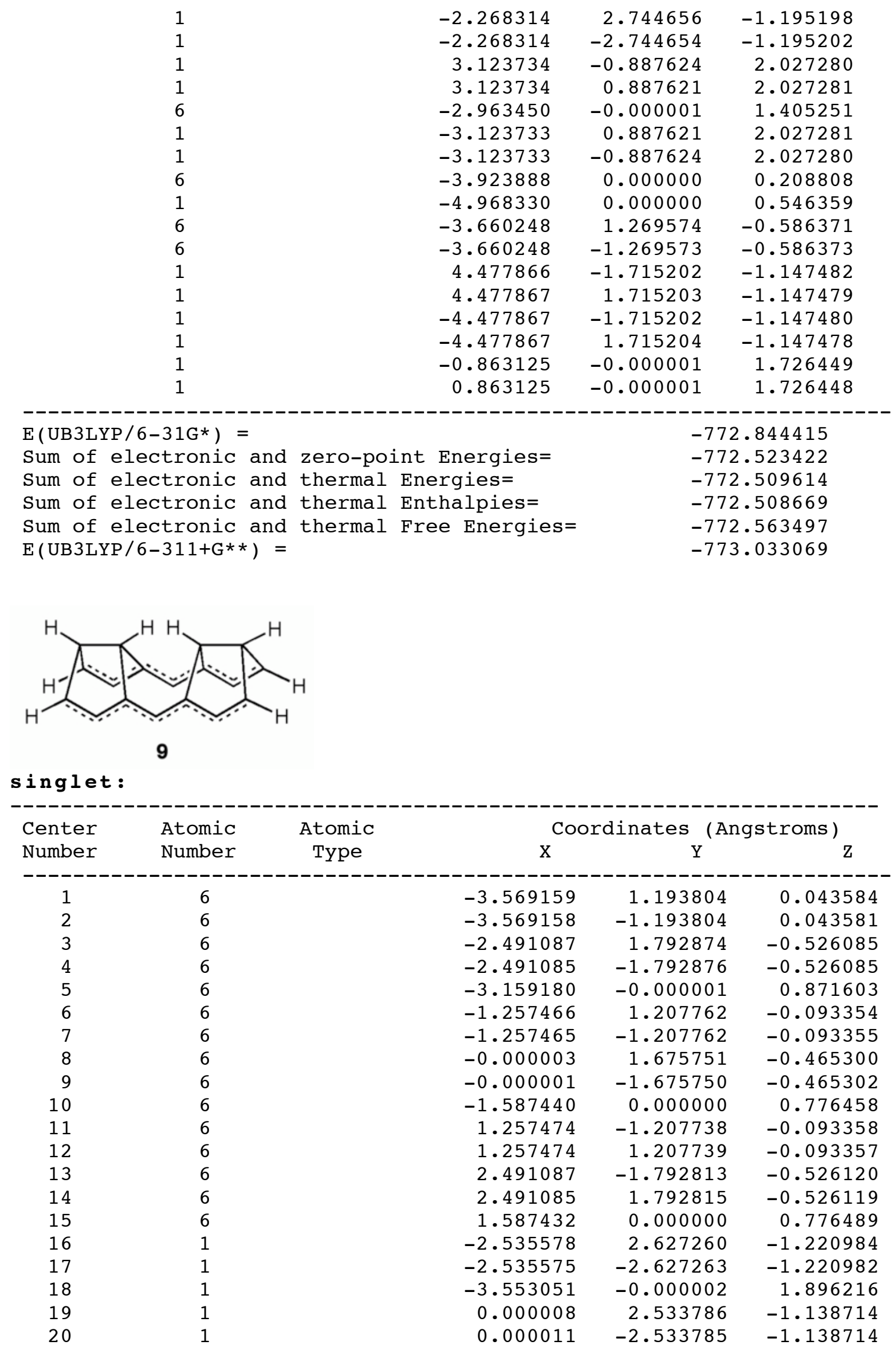




\begin{tabular}{rrrrr}
21 & 1 & 2.535590 & -2.627156 & -1.221069 \\
22 & 1 & 2.535588 & 2.627158 & -1.221069 \\
23 & 6 & 3.159169 & 0.000001 & 0.871654 \\
24 & 1 & 3.553041 & 0.000002 & 1.896265 \\
25 & 6 & 3.569161 & -1.193766 & 0.043591 \\
26 & 6 & 3.569161 & 1.193764 & 0.043586 \\
27 & 1 & -4.606607 & 1.461838 & -0.127701 \\
28 & 1 & -4.606605 & -1.461839 & -0.127705 \\
29 & 1 & 4.606610 & 1.461786 & -0.127705 \\
30 & 1 & 4.606611 & -1.461789 & -0.127697 \\
31 & 1 & -1.099996 & 0.000000 & 1.753593 \\
32 & 1 & 1.099961 & 0.000001 & 1.753611 \\
\hline
\end{tabular}

$\mathrm{HF}=-694.2296114$

$\mathrm{ZPE}=0.262441$

(single point at B3LYP/6-311+G**, E=-694.39829)

triplet:

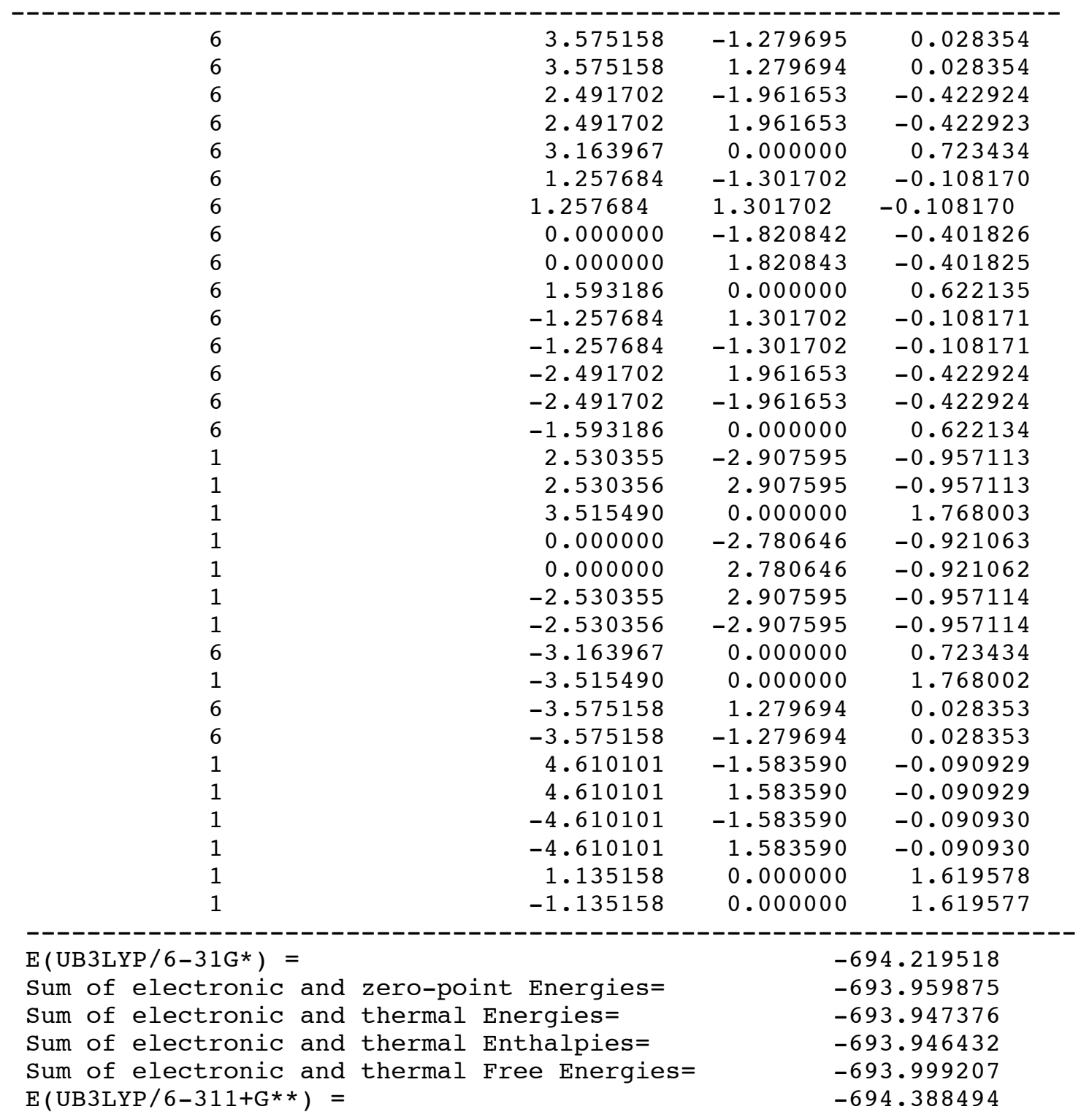




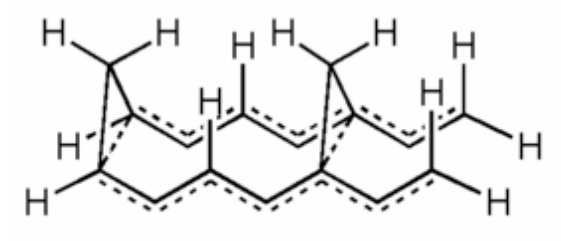

10

singlet :

\begin{tabular}{|c|c|c|c|c|c|}
\hline \multirow{2}{*}{$\begin{array}{l}\text { Center } \\
\text { Number }\end{array}$} & \multirow{2}{*}{$\begin{array}{l}\text { Atomic } \\
\text { Number }\end{array}$} & \multirow{2}{*}{$\begin{array}{c}\text { Atomic } \\
\text { Type }\end{array}$} & \multicolumn{3}{|c|}{ Coordinates (Angstroms) } \\
\hline & & & $\mathrm{X}$ & $\mathrm{Y}$ & Z \\
\hline 1 & 6 & & -3.547092 & 1.149622 & -0.163153 \\
\hline 2 & 6 & & -3.547048 & -1.149626 & -0.163214 \\
\hline 3 & 6 & & -2.330336 & 1.703227 & -0.461308 \\
\hline 4 & 6 & & -2.330282 & -1.703217 & -0.461360 \\
\hline 5 & 6 & & -3.779325 & -0.000029 & 0.788040 \\
\hline 6 & 6 & & -1.112218 & 1.378268 & 0.189910 \\
\hline 7 & 6 & & -1.112179 & -1.378290 & 0.189904 \\
\hline 8 & 6 & & 0.159533 & 1.686222 & -0.305844 \\
\hline 9 & 6 & & 0.159582 & -1.686254 & -0.305818 \\
\hline 10 & 6 & & 1.375883 & -1.179733 & 0.148653 \\
\hline 11 & 6 & & 1.375847 & 1.179710 & 0.148611 \\
\hline 12 & 6 & & 2.583080 & -1.753355 & -0.417896 \\
\hline 13 & 6 & & 2.583031 & 1.753360 & -0.417934 \\
\hline 14 & 6 & & 1.453001 & 0.000005 & 1.107107 \\
\hline 15 & 1 & & -2.271306 & 2.367526 & -1.324640 \\
\hline 16 & 1 & & -2.271231 & -2.367497 & -1.324706 \\
\hline 17 & 1 & & -4.800530 & -0.000054 & 1.181136 \\
\hline 18 & 1 & & 0.200379 & 2.349743 & -1.172343 \\
\hline 19 & 1 & & 0.200444 & -2.349764 & -1.172325 \\
\hline 20 & 1 & & 2.414874 & -2.395127 & -1.284054 \\
\hline 21 & 1 & & 2.414819 & 2.395101 & -1.284114 \\
\hline 22 & 6 & & 3.850211 & -1.636107 & 0.025047 \\
\hline 23 & 6 & & 3.850155 & 1.636181 & 0.025045 \\
\hline 24 & 1 & & -4.402889 & 1.434874 & -0.771191 \\
\hline 25 & 1 & & -4.402834 & -1.434878 & -0.771269 \\
\hline 26 & 1 & & 4.662150 & 2.151120 & -0.479230 \\
\hline 27 & 1 & & 4.662213 & -2.151028 & -0.479233 \\
\hline 28 & 1 & & -3.098316 & -0.000039 & 1.643599 \\
\hline 29 & 1 & & 2.382699 & 0.000025 & 1.680461 \\
\hline 30 & 1 & & 0.639983 & 0.000004 & 1.837958 \\
\hline 31 & 1 & & -1.179447 & -0.932849 & 1.170901 \\
\hline 32 & 1 & & -1.179458 & 0.932809 & 1.170900 \\
\hline 33 & 1 & & 4.118666 & 1.074732 & 0.911720 \\
\hline 34 & 1 & & 4.118722 & -1.074600 & 0.911683 \\
\hline
\end{tabular}

$\mathrm{HF}=-620.3895917$

$\mathrm{ZPE}=0.291629$

(single point at B3LYP/6-311+G**, E=-620.55541)

triplet: 


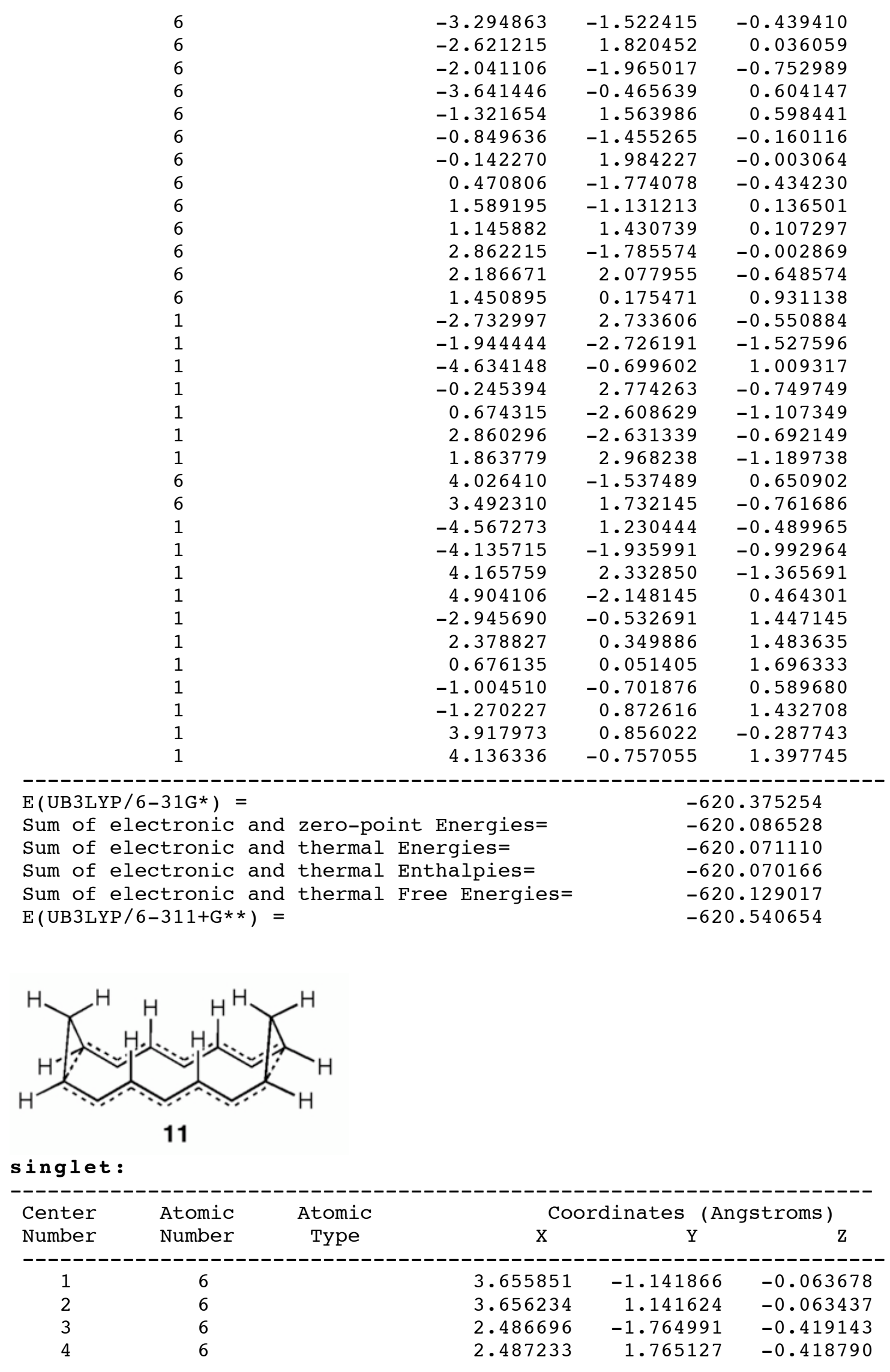




\begin{tabular}{|c|c|c|c|c|}
\hline 5 & 6 & 3.797440 & -0.000253 & 0.911208 \\
\hline 6 & 6 & 1.222972 & -1.564468 & 0.186448 \\
\hline 7 & 6 & 1.223346 & 1.564218 & 0.186292 \\
\hline 8 & 6 & -0.000216 & -1.953768 & -0.355702 \\
\hline 9 & 6 & 0.000136 & 1.954021 & -0.355540 \\
\hline 10 & 6 & -1.222880 & 1.564420 & 0.186417 \\
\hline 11 & 6 & -1.223258 & -1.564646 & 0.186525 \\
\hline 12 & 6 & -2.486897 & 1.765730 & -0.418674 \\
\hline 13 & 6 & -2.487252 & -1.765569 & -0.418714 \\
\hline 14 & 6 & -3.797299 & 0.000198 & 0.910736 \\
\hline 15 & 6 & -3.655914 & 1.142408 & -0.063510 \\
\hline 16 & 6 & -3.656180 & -1.142082 & -0.063505 \\
\hline 17 & 1 & 4.550099 & -1.374860 & -0.638007 \\
\hline 18 & 1 & 4.550582 & 1.374660 & -0.637576 \\
\hline 19 & 1 & 2.516032 & -2.427415 & -1.285138 \\
\hline 20 & 1 & 2.516869 & 2.428212 & -1.284271 \\
\hline 21 & 1 & 4.775153 & -0.000464 & 1.404161 \\
\hline 22 & 1 & 3.031705 & -0.000185 & 1.690505 \\
\hline 23 & 1 & 1.190250 & -1.044453 & 1.138757 \\
\hline 24 & 1 & 1.190569 & 1.042944 & 1.137848 \\
\hline 25 & 1 & -0.000188 & -2.486931 & -1.307725 \\
\hline 26 & 1 & 0.000027 & 2.487557 & -1.307357 \\
\hline 27 & 1 & -1.190132 & 1.043242 & 1.138052 \\
\hline 28 & 1 & -1.190477 & -1.044181 & 1.138587 \\
\hline 29 & 1 & -2.516387 & 2.429101 & -1.283941 \\
\hline 30 & 1 & -2.516866 & -2.428741 & -1.284134 \\
\hline 31 & 1 & -4.774977 & 0.000311 & 1.403826 \\
\hline 32 & 1 & -3.031629 & 0.000090 & 1.690102 \\
\hline 33 & 1 & -4.550202 & 1.375750 & -0.637628 \\
\hline 34 & 1 & -4.550491 & -1.375258 & -0.637653 \\
\hline
\end{tabular}

$\mathrm{HF}=-620.400351$

$\mathrm{ZPE}=0.291813$

(single point at B3LYP/6-311+G**, E=--620.56570)

triplet:

$\begin{array}{lrrr}6 & 3.612834 & -1.258893 & -0.142582 \\ 6 & 3.612924 & 1.259011 & -0.142311 \\ 6 & 2.497415 & -2.017622 & -0.348159 \\ 6 & 2.497484 & 2.017711 & -0.347917 \\ 6 & 3.706426 & -0.000038 & 0.701547 \\ 6 & 1.216169 & -1.793166 & 0.235828 \\ 6 & 1.216161 & 1.792960 & 0.235768 \\ 6 & -0.000010 & -2.291703 & -0.228156 \\ 6 & 0.000003 & 2.291706 & -0.228047 \\ 6 & -1.216144 & 1.792963 & 0.235795 \\ 6 & -1.216173 & -1.793163 & 0.235857 \\ 6 & -2.497480 & 2.017715 & -0.347864 \\ 6 & -2.497438 & -2.017619 & -0.348094 \\ 6 & -3.706409 & -0.000024 & 0.701635 \\ 6 & -3.612917 & 1.259019 & -0.142233 \\ 6 & -3.612847 & -1.258884 & -0.142489 \\ 1 & 4.513434 & -1.521318 & -0.695233 \\ 1 & 4.513623 & 1.521652 & -0.694697 \\ 1 & 2.572041 & -2.833054 & -1.068772 \\ 1 & 2.572196 & 2.833375 & -1.068257 \\ 1 & 4.672681 & -0.000122 & 1.225077\end{array}$




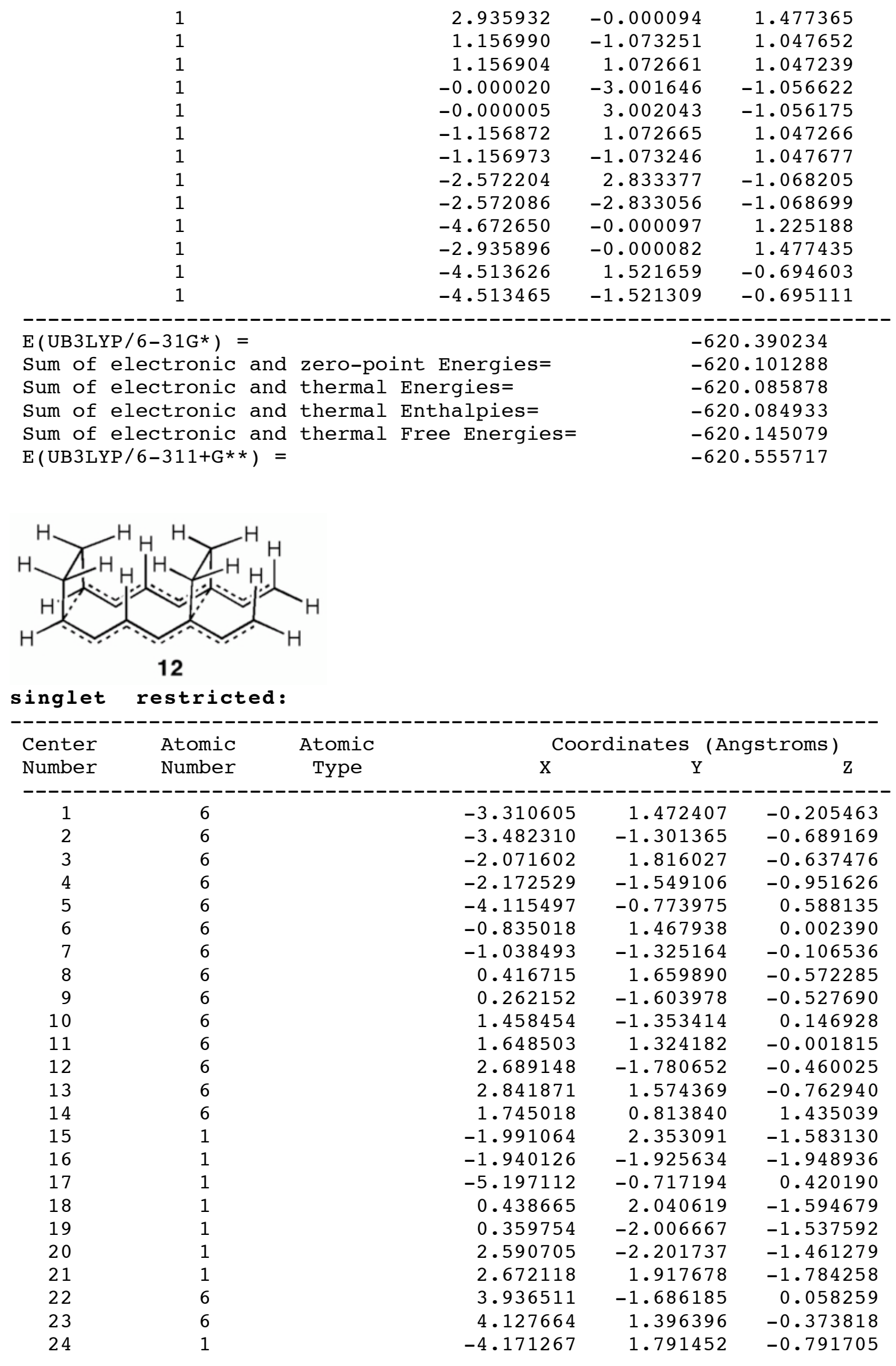




$\begin{array}{rrrrr}25 & 1 & -4.184797 & -1.551044 & -1.482405 \\ 26 & 1 & 4.945104 & 1.606964 & -1.056320 \\ 27 & 1 & 4.798421 & -2.044923 & -0.495007 \\ 28 & 1 & -3.980166 & -1.483170 & 1.419737 \\ 29 & 1 & 2.738637 & 1.042247 & 1.829755 \\ 30 & 1 & 1.044572 & 1.368760 & 2.071476 \\ 31 & 1 & -1.221461 & -1.053743 & 0.926644 \\ 32 & 1 & -0.874744 & 1.140875 & 1.034387 \\ 33 & 1 & 4.408519 & 1.095332 & 0.628401 \\ 34 & 1 & 4.130292 & -1.310839 & 1.057672 \\ 35 & 6 & -3.606177 & 0.627504 & 1.009702 \\ 36 & 6 & 1.468903 & -0.713235 & 1.529134 \\ 37 & 1 & 2.220706 & -1.200137 & 2.161569 \\ 38 & 1 & 0.510653 & -0.894735 & 2.025294 \\ 39 & 1 & -2.712071 & 0.528262 & 1.630811 \\ 40 & 1 & -4.361581 & 1.111656 & 1.644914 \\ ----1.6314 & & & \end{array}$

$\mathrm{HF}=-699.0077651$

$\mathrm{ZPE}=0.348882$

singlet unrestricted:

$\begin{array}{lrrr}6 & 3.217901 & -1.632474 & -0.066957 \\ 6 & 3.557516 & 1.324649 & -0.785355 \\ 6 & 1.978787 & -2.063070 & -0.425338 \\ 6 & 2.258450 & 1.678104 & -1.011953 \\ 6 & 4.192506 & 0.673502 & 0.427715 \\ 6 & 0.734001 & -1.680963 & 0.171968 \\ 6 & 1.132090 & 1.475704 & -0.164984 \\ 6 & -0.495237 & -1.892109 & -0.436798 \\ 6 & -0.174789 & 1.802846 & -0.530872 \\ 6 & -1.355790 & 1.531808 & 0.169355 \\ 6 & -1.756754 & -1.459334 & 0.000184 \\ 6 & -2.590103 & 2.066545 & -0.342903 \\ 6 & -2.884274 & -1.784084 & -0.832405 \\ 1 & -1.959157 & -0.727557 & 1.317119 \\ 1 & 1.908778 & -2.727640 & -1.287469 \\ 1 & 2.046011 & 2.147016 & -1.974065 \\ 1 & 5.228686 & 0.432267 & 0.160694 \\ 1 & -0.475426 & -2.417451 & -1.393171 \\ 1 & -0.290938 & 2.324131 & -1.483025 \\ 1 & -2.512024 & 2.589059 & -1.297065 \\ 6 & -2.633411 & -2.331369 & -1.742135 \\ 6 & -3.811086 & 2.009853 & 0.241830 \\ 1 & -4.197535 & -1.504767 & -0.633820 \\ 1 & 4.069803 & -2.003020 & -0.636879 \\ 1 & 4.259992 & 1.574580 & -1.578919 \\ 1 & -4.940886 & -1.818568 & -1.360060 \\ 1 & -4.677106 & 2.465720 & -0.227380 \\ 1 & 4.266868 & 1.403129 & 1.250025 \\ 1 & -3.030359 & -0.671682 & 1.518947 \\ 1 & -1.539174 & -1.333376 & 2.132484 \\ 1 & 1.312044 & 1.042314 & 0.810359 \\ 1 & 0.761459 & -1.171409 & 1.129011 \\ 1 & -4.570408 & -0.962773 & 0.228161 \\ 6 & -3.978964 & 1.528692 & 1.200545 \\ 6 & -1.357383 & -0.618212 & 0.995536 \\ 6 & 0.710233 & 1.442834\end{array}$




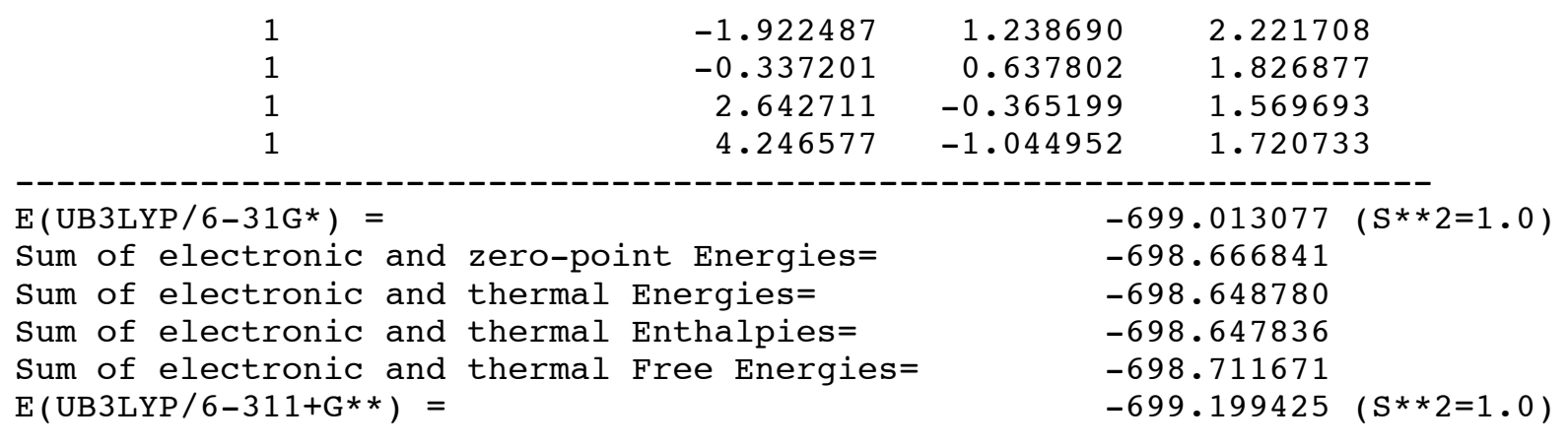

triplet:

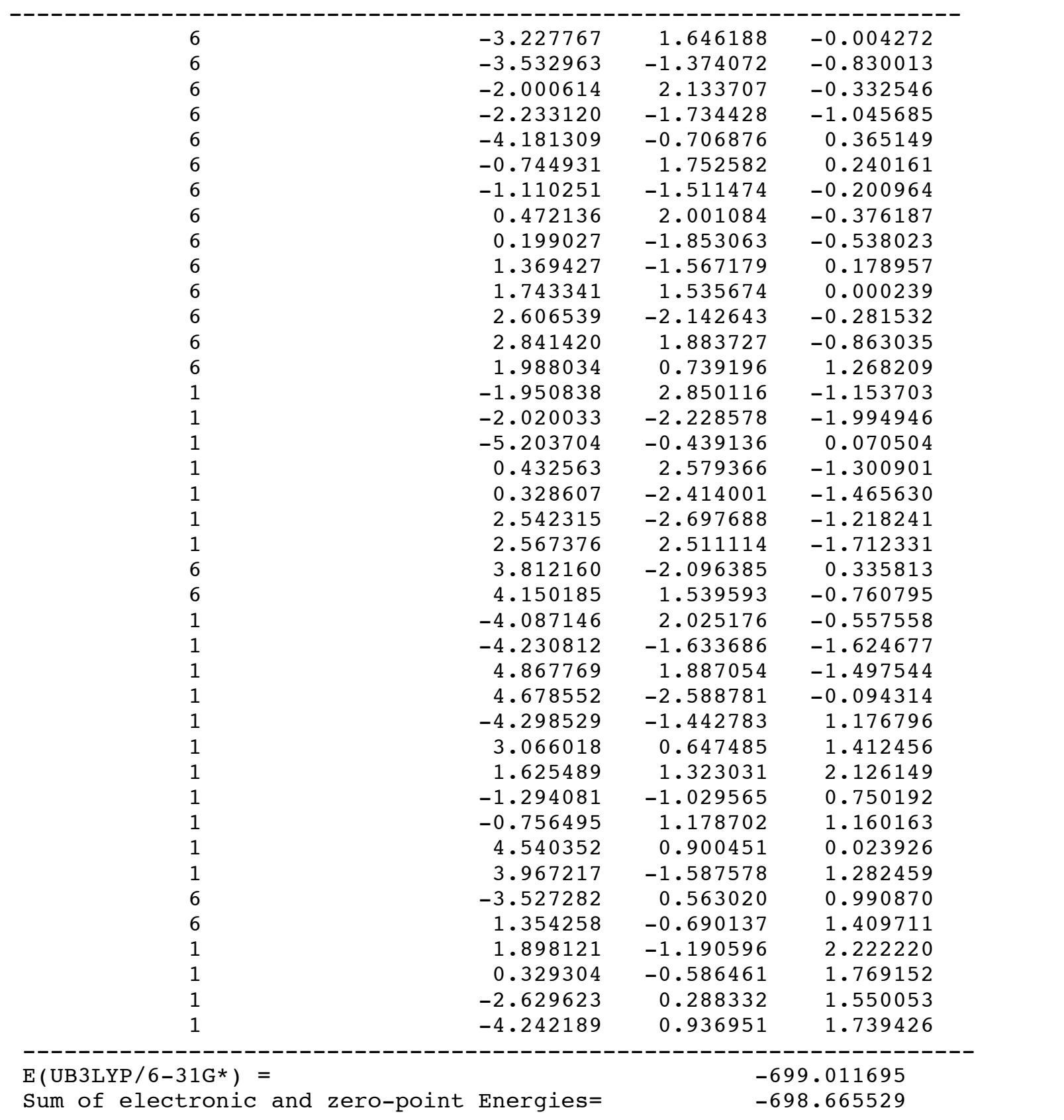


Sum of electronic and thermal Energies=

$-698.647441$

Sum of electronic and thermal Enthalpies=

Sum of electronic and thermal Free Energies=

$-698.646496$

$-698.711626$

$\mathrm{E}(\mathrm{UB} 3 \mathrm{LYP} / 6-311+\mathrm{G} *$ *) $=$

$-699.197871$

Singlet 13

\begin{tabular}{|c|c|c|c|}
\hline 6 & -2.121727 & 1.790650 & -0.190790 \\
\hline 6 & -0.855513 & 1.247408 & -0.032929 \\
\hline 6 & -0.668416 & 0.001092 & 0.837405 \\
\hline 6 & -0.860154 & -1.244489 & -0.032889 \\
\hline 6 & 0.263635 & 1.770291 & -0.675311 \\
\hline 6 & 0.729044 & -0.001622 & 1.482643 \\
\hline 6 & 0.257004 & -1.770503 & -0.676351 \\
\hline 6 & -2.128015 & -1.784010 & -0.189725 \\
\hline 6 & 1.669384 & 1.290828 & -0.464489 \\
\hline 6 & 1.793722 & -0.003144 & 0.372123 \\
\hline 6 & 1.664466 & -1.295929 & -0.465679 \\
\hline 1 & -2.985223 & 1.382180 & 0.324281 \\
\hline 1 & -2.290291 & 2.644517 & -0.841152 \\
\hline 1 & -1.436524 & 0.002642 & 1.619200 \\
\hline 1 & 0.129879 & 2.633224 & -1.326591 \\
\hline 1 & 0.846074 & 0.882272 & 2.122598 \\
\hline 1 & 0.842857 & -0.886398 & 2.121957 \\
\hline 1 & 0.120125 & -2.632573 & -1.328122 \\
\hline 1 & -2.989887 & -1.373180 & 0.326202 \\
\hline 1 & -2.299631 & -2.637254 & -0.840109 \\
\hline 1 & 2.234945 & 2.092923 & 0.041556 \\
\hline 1 & 2.175928 & 1.162716 & -1.433886 \\
\hline 1 & 2.792647 & -0.005279 & 0.825643 \\
\hline 1 & 2.227367 & -2.100446 & 0.039504 \\
\hline 1 & 2.171149 & -1.168782 & -1.435126 \\
\hline
\end{tabular}

Electronic energy (UB3LYP/6-31G*//UB3LYP/6-31G*) $=-427.494189(\mathrm{~S} * * 2=1.02)$

Sum of electronic and zero-point Energies= -427.277155

Sum of electronic and thermal Energies= $\quad-427.267531$

Sum of electronic and thermal Enthalpies= $\quad-427.266587$

Sum of electronic and thermal Free Energies= $\quad-427.311234$

Electronic energy (UB3LYP/6-311+G**//UB3LYP/6-31G*) $=-427.605907 \quad(\mathrm{~S} * * 2=1.01$ )

Triplet 13

\begin{tabular}{|c|c|c|c|}
\hline 6 & -2.114701 & 1.828093 & -0.193438 \\
\hline 6 & -0.856788 & 1.261398 & -0.045574 \\
\hline 6 & -0.683779 & 0.000560 & 0.809495 \\
\hline 6 & -0.859029 & -1.259982 & -0.045536 \\
\hline 6 & 0.272182 & 1.799237 & -0.657320 \\
\hline 6 & 0.705750 & -0.000717 & 1.474357 \\
\hline 6 & 0.268967 & -1.799582 & -0.657538 \\
\hline 6 & -2.117858 & -1.824715 & -0.193074 \\
\hline 6 & 1.671652 & 1.295655 & -0.458652 \\
\hline 6 & 1.784075 & -0.001532 & 0.377010 \\
\hline 6 & 1.669278 & -1.298191 & -0.459192 \\
\hline 1 & -2.990224 & 1.407997 & 0.291422 \\
\hline 1 & -2.264673 & 2.709960 & -0.810052 \\
\hline 1 & -1.460718 & 0.001269 & 1.582559 \\
\hline 1 & 0.152414 & 2.689898 & -1.272959 \\
\hline 1 & 0.813520 & 0.883540 & 2.115265 \\
\hline 1 & 0.811951 & -0.885290 & 2.115091 \\
\hline 1 & 0.147678 & -2.690038 & -1.273175 \\
\hline 1 & -2.992598 & -1.403287 & 0.292046 \\
\hline
\end{tabular}




\begin{tabular}{crrr}
1 & -2.269368 & -2.706327 & -0.809679 \\
1 & 2.252051 & 2.089118 & 0.043949 \\
1 & 2.169318 & 1.162674 & -1.431853 \\
1 & 2.776748 & -0.002534 & 0.843897 \\
1 & 2.248653 & -2.092785 & 0.042794 \\
\hline-166758 & -1.165537 & -1.432537 \\
\hline Electronic energy (UB3LYP/6-31g* opt)= & -427.492668 \\
Sum of electronic and zero-point Energies= & -427.275759 \\
Sum of electronic and thermal Energies= & -427.266099 \\
Sum of electronic and thermal Enthalpies= & -427.265155 \\
Sum of electronic and thermal Free Energies= & -427.311092 \\
Electronic energy (UB3LYP/6-311+g**//opt)= & -427.604114
\end{tabular}

Singlet 14

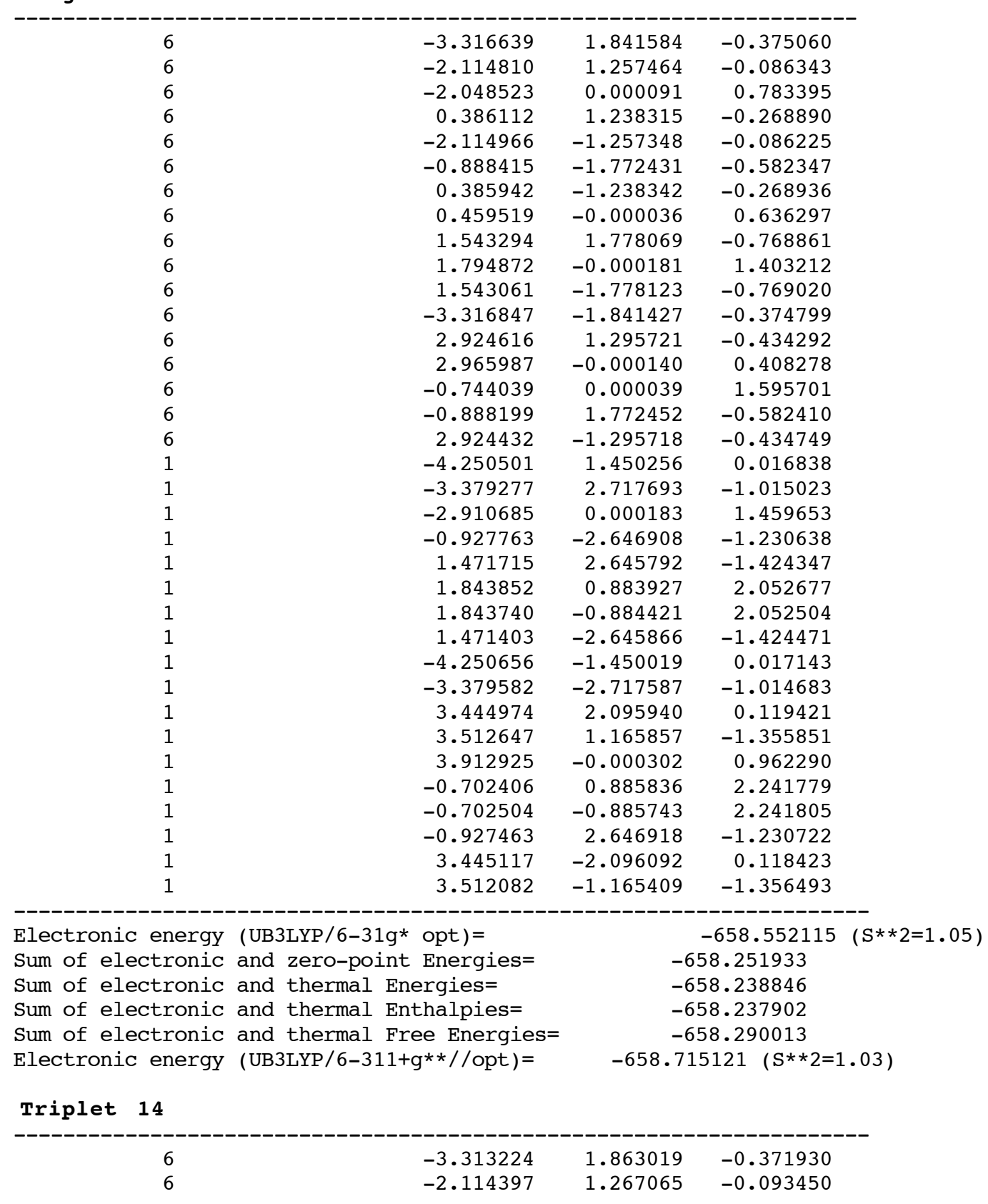




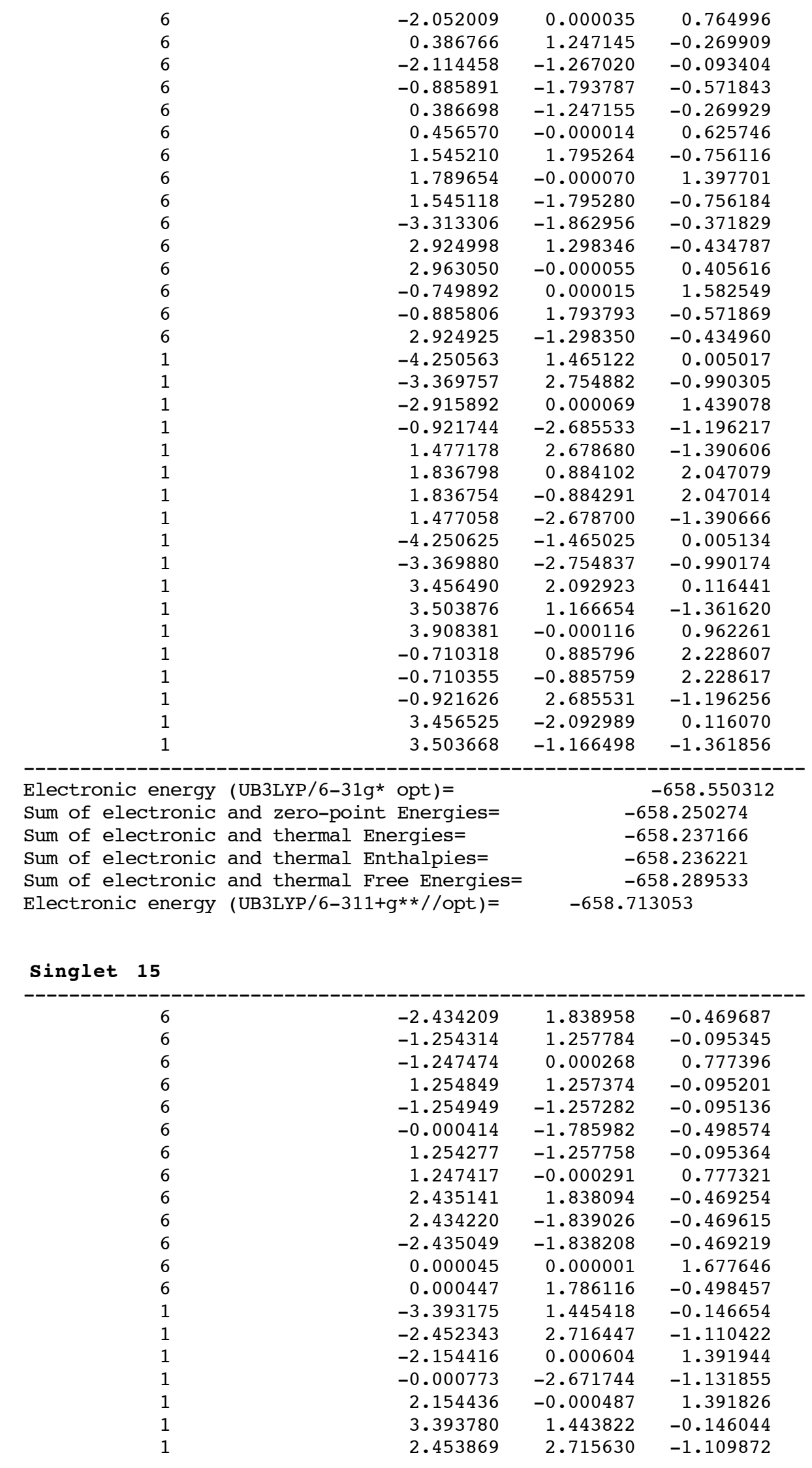




$\begin{array}{rrrr}1 & 3.393105 & -1.445411 & -0.146303 \\ 1 & 2.452472 & -2.716443 & -1.110398 \\ 1 & -3.393864 & -1.444464 & -0.145989 \\ 1 & -2.453461 & -2.715674 & -1.109973 \\ 1 & 0.000190 & 0.886076 & 2.323127 \\ 1 & -0.000222 & -0.886058 & 2.323162 \\ 1 & 0.000488 & 2.671987 & -1.131602\end{array}$

Electronic energy (UB3LYP/6-31g* opt) = Sum of electronic and zero-point Energies= Sum of electronic and thermal Energies= Sum of electronic and thermal Enthalpies= Sum of electronic and thermal Free Energies= Electronic energy (UB3LYP/6-311+g**//opt) =
$-503.693794(\mathrm{~S} * * 2=1.07)$

$$
\begin{aligned}
& -503.466832 \\
& -503.455591 \\
& -503.454647 \\
& -503.502957
\end{aligned}
$$

$-503.824491(S * * 2=1.04)$

Triplet 15

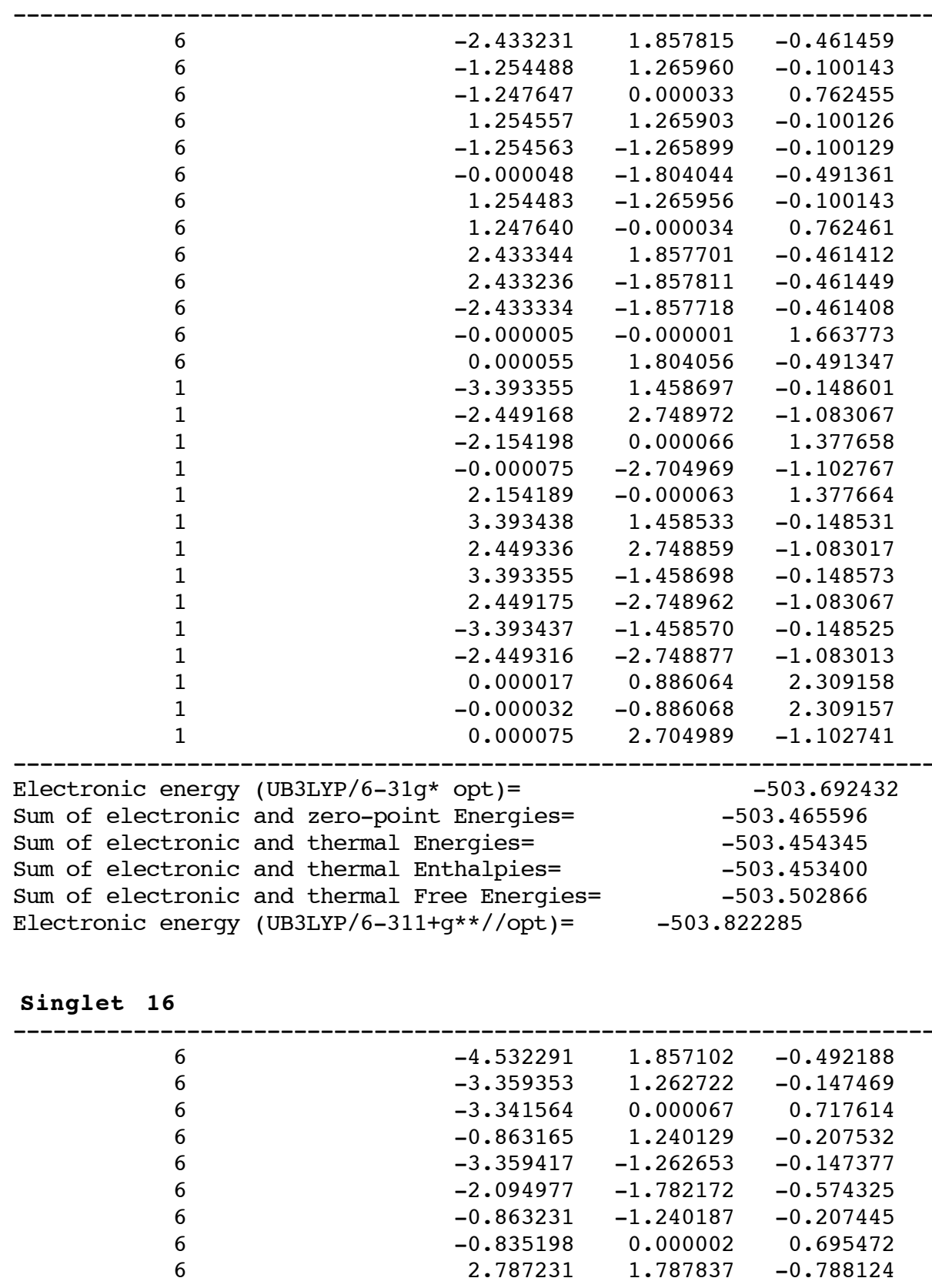




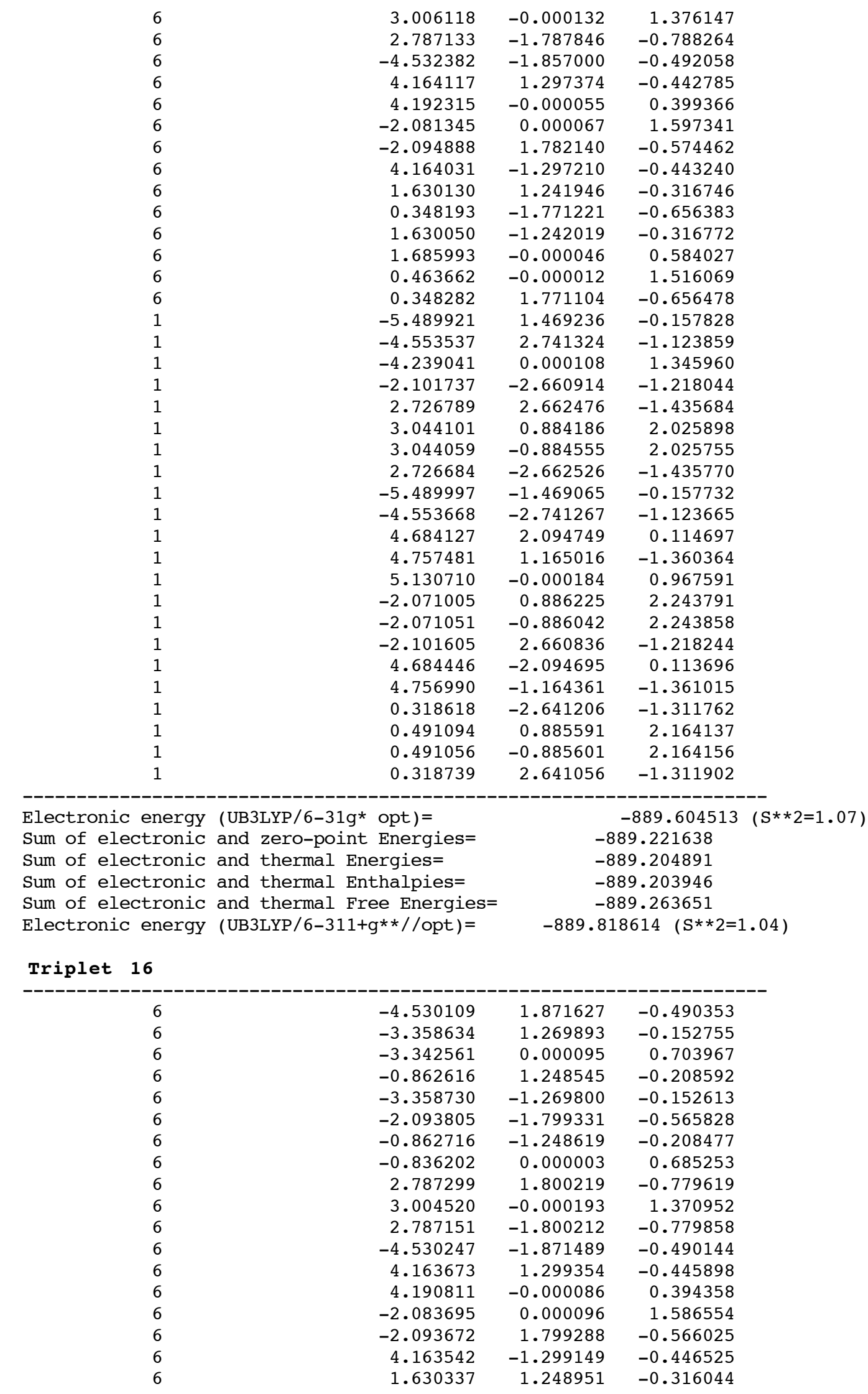




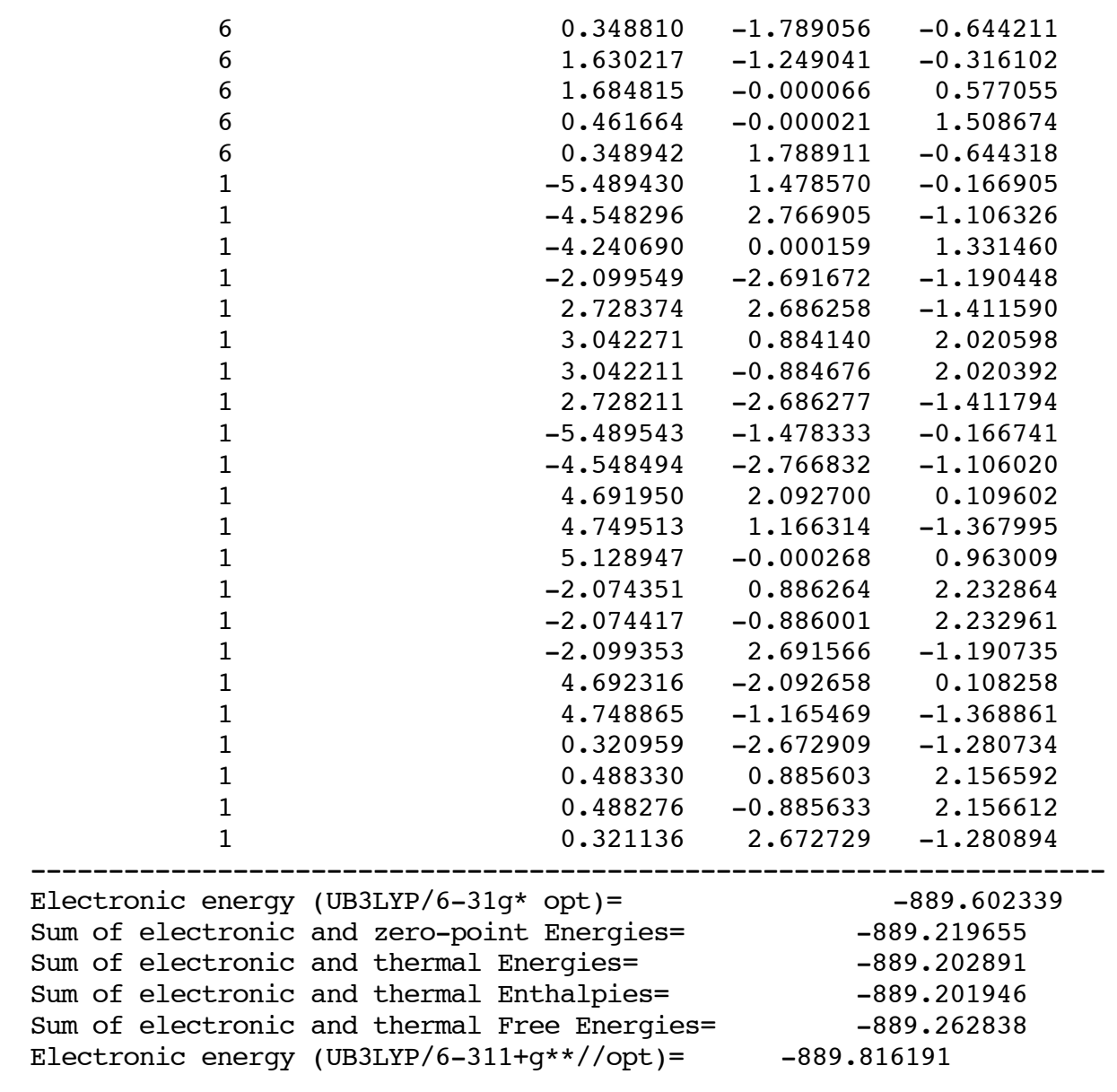

Singlet 17

$\begin{array}{rrrr}6 & 5.810852 & 0.000156 & -0.754352 \\ 6 & 5.128673 & 1.327640 & -1.161535 \\ 6 & 5.129398 & -1.328071 & -1.160421 \\ 6 & 3.374054 & 0.000172 & 1.035019 \\ 6 & 2.938522 & 1.252242 & 0.287003 \\ 6 & 2.938577 & -1.251908 & 0.287078 \\ 6 & 3.784593 & 1.786182 & -0.640399 \\ 6 & 3.784683 & -1.785917 & -0.640227 \\ 6 & 1.599272 & -1.792444 & 0.377280 \\ 6 & 1.599264 & 1.792762 & 0.377256 \\ 6 & -3.100274 & -1.788462 & -0.202600 \\ 6 & -3.100375 & 1.788158 & -0.202732 \\ 6 & -5.057209 & -0.000221 & -0.062364 \\ 6 & -4.424118 & 1.310570 & -0.549254 \\ 6 & -4.423861 & -1.310809 & -0.549527 \\ 6 & -5.161545 & 2.068989 & -1.404196 \\ 6 & -5.160881 & -2.068852 & -1.405141 \\ 6 & 0.505407 & 0.000122 & 1.801630 \\ 6 & 0.441958 & 1.256460 & 0.943193 \\ 6 & 0.441957 & -1.256288 & 0.943275 \\ 6 & -2.435925 & -0.000034 & 1.420339 \\ 6 & -2.115299 & 1.248649 & 0.615425 \\ 6 & -2.115292 & -1.248813 & 0.615561 \\ 6 & -0.805105 & 1.792715 & 0.531713 \\ 1 & -0.805064 & -1.792864 & 0.532027 \\ 1 & 6.774077 & 0.000166 & -1.283151 \\ & 6.084449 & 0.000640 & 0.305362\end{array}$




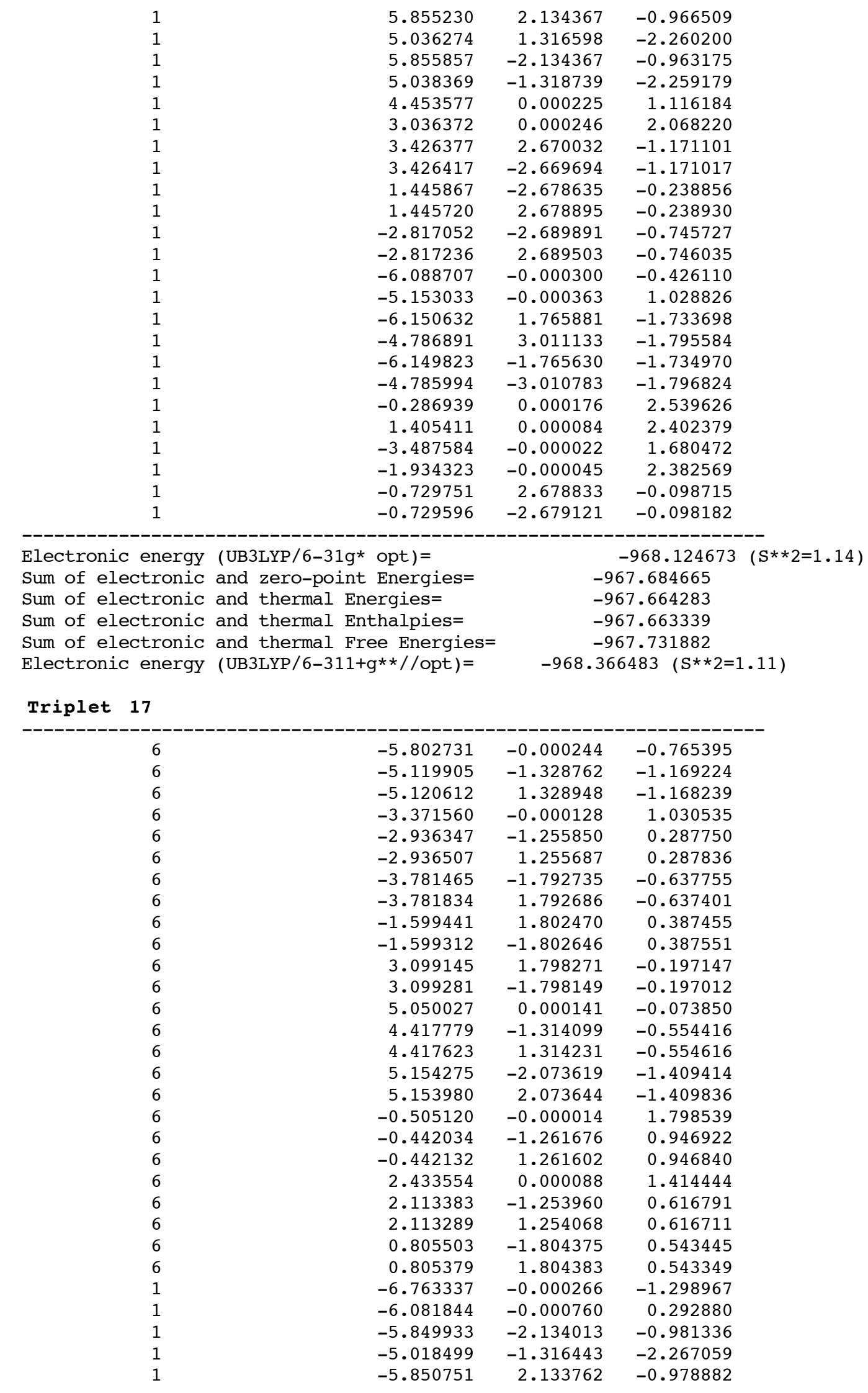




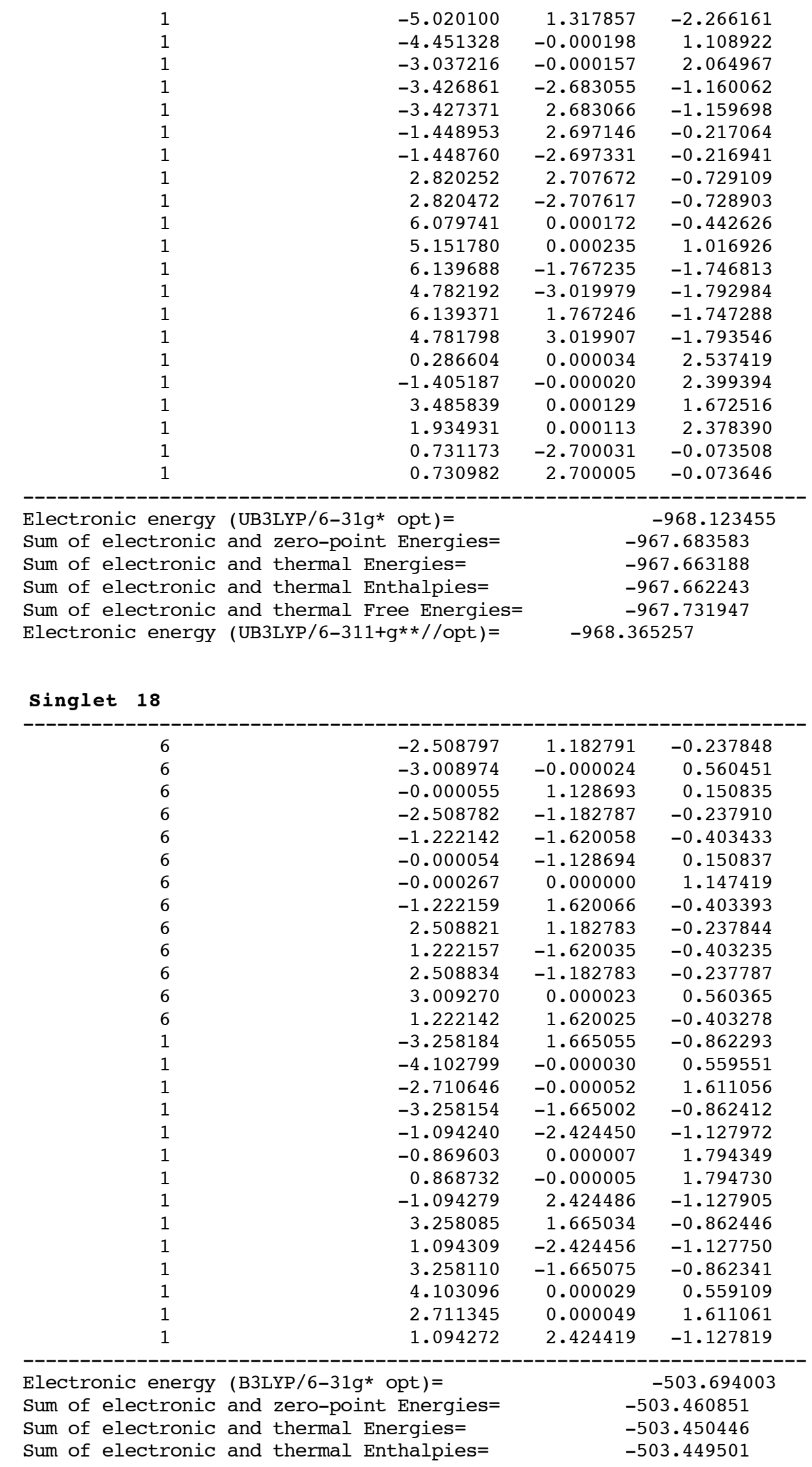


Sum of electronic and thermal Free Energies= Electronic energy (B3LYP/6-311+g**//opt $)=$
$-503.495791$

$-503.824878$

Triplet 18

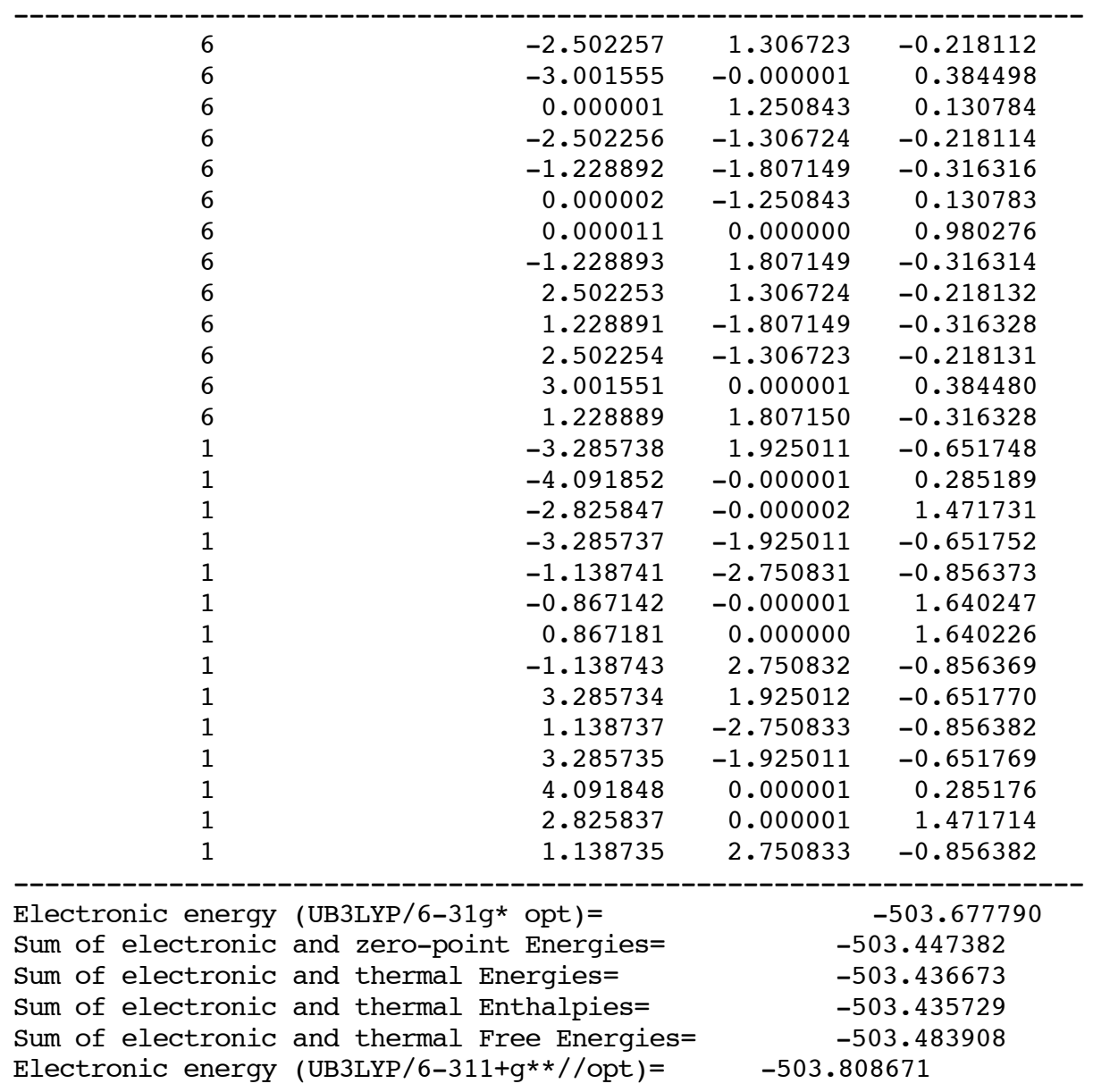

exo-syn-Tricyclo[5.5.1.03,5]trideca-1,6,8,11-tetraene (end-closed analog of 18 )

$\begin{array}{rrrr}6 & 2.476883 & -1.301654 & -0.218748 \\ 6 & 3.000408 & 0.000002 & 0.387565 \\ 6 & -0.026393 & -1.192940 & 0.160973 \\ 6 & 2.476879 & 1.301655 & -0.218755 \\ 6 & 1.223627 & 1.795861 & -0.296693 \\ 6 & -0.026395 & 1.192942 & 0.160978 \\ 6 & 0.044978 & 0.000000 & 1.088718 \\ 6 & 1.223631 & -1.795861 & -0.296690 \\ 6 & -2.483806 & -0.801815 & -0.371899 \\ 6 & -1.204979 & 1.533890 & -0.422170 \\ 6 & -2.483809 & 0.801816 & -0.371889 \\ 6 & -3.061368 & -0.000007 & 0.767099 \\ 1 & -1.204975 & -1.533886 & -0.422179 \\ 1 & 3.248467 & -1.904521 & -0.694997 \\ 1 & 4.085579 & 0.000004 & 0.242213 \\ 1 & 2.873713 & 0.000004 & 1.478674 \\ 1 & 3.248460 & 1.904517 & -0.695014 \\ 1 & 1.104426 & 2.731050 & -0.845009 \\ 1 & 0.953881 & -0.000001 & 1.683027 \\ & -0.770368 & -0.000002 & 1.806675\end{array}$




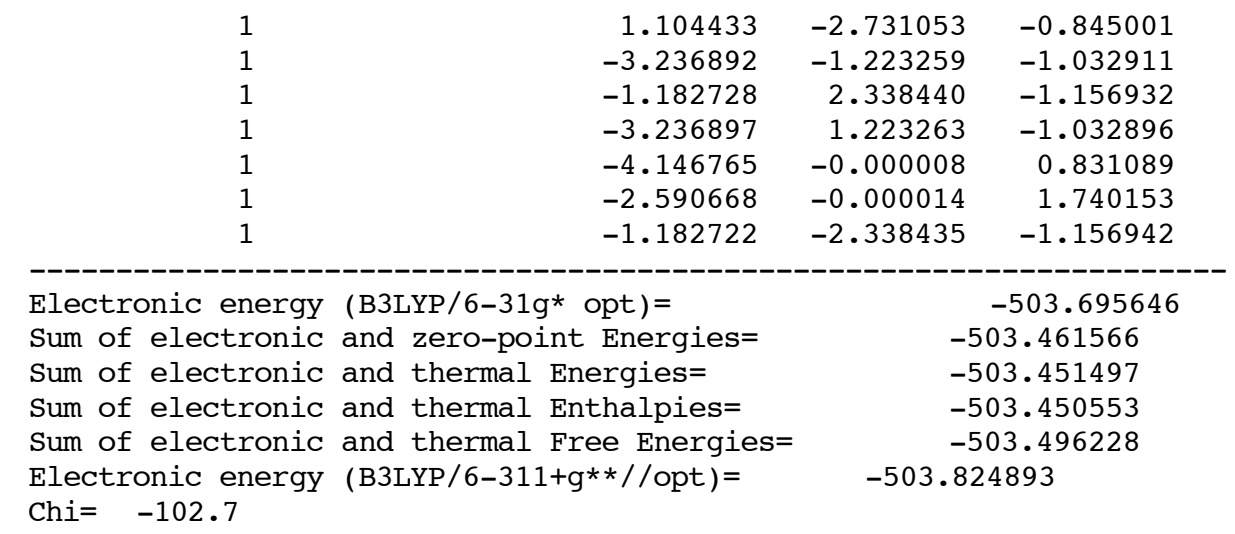

\section{Singlet 19}

\begin{tabular}{|c|c|c|c|}
\hline 6 & -2.422612 & 1.268456 & -0.196129 \\
\hline 6 & -2.479625 & 0.000002 & -1.025170 \\
\hline 6 & 0.009867 & 1.042838 & 0.471676 \\
\hline 6 & -2.423046 & -1.268261 & -0.195810 \\
\hline 6 & -1.325815 & -1.704946 & 0.430973 \\
\hline 6 & 0.009682 & -1.042547 & 0.471746 \\
\hline 6 & 0.226409 & 0.000090 & 1.532006 \\
\hline 6 & -1.325236 & 1.705135 & 0.430384 \\
\hline 6 & 2.320042 & 1.118910 & -0.561221 \\
\hline 6 & 1.017494 & -1.536883 & -0.389428 \\
\hline 6 & 2.319410 & -1.119214 & -0.561994 \\
\hline 6 & 3.018331 & -0.000603 & 0.159117 \\
\hline 6 & 1.018210 & 1.537049 & -0.388947 \\
\hline 1 & -3.340520 & 1.847649 & -0.103539 \\
\hline 1 & -3.387781 & 0.000124 & -1.636780 \\
\hline 1 & -1.626265 & -0.000239 & -1.715442 \\
\hline 1 & -3.341195 & -1.847015 & -0.102844 \\
\hline 1 & -1.390098 & -2.639801 & 0.994553 \\
\hline 1 & -0.567276 & 0.000194 & 2.280057 \\
\hline 1 & 1.189608 & 0.000233 & 2.034617 \\
\hline 1 & -1.389326 & 2.640675 & 0.992906 \\
\hline 1 & 2.862577 & 1.550898 & -1.399356 \\
\hline 1 & 0.672195 & -2.293293 & -1.093346 \\
\hline 1 & 2.861673 & -1.551211 & -1.400298 \\
\hline 1 & 4.088395 & -0.000782 & -0.063695 \\
\hline 1 & 2.915942 & -0.001078 & 1.243031 \\
\hline 1 & 0.673397 & 2.293485 & -1.093084 \\
\hline
\end{tabular}

Electronic energy (B3LYP/6-31g* opt)=

Sum of electronic and zero-point Energies=

Sum of electronic and thermal Energies=

Sum of electronic and thermal Enthalpies=

Sum of electronic and thermal Free Energies=

Electronic energy (B3LYP/6-311+g**//opt) $=$

$-503.670818$

$-503.440059$

$-503.429737$

$-503.428793$

$-503.475366$

$-503.802570$

\section{Singlet 20}

$\begin{array}{rrrr}6 & 1.277311 & 1.166992 & 0.331222 \\ 6 & 1.507245 & 0.000005 & 1.263642 \\ 6 & 1.277311 & -1.166991 & 0.331234 \\ 6 & 4.316786 & 0.000004 & 0.076422 \\ 6 & 3.671578 & -1.233240 & -0.531680 \\ 6 & 3.671577 & 1.233241 & -0.531693 \\ 6 & 2.394302 & -1.688835 & -0.420800\end{array}$




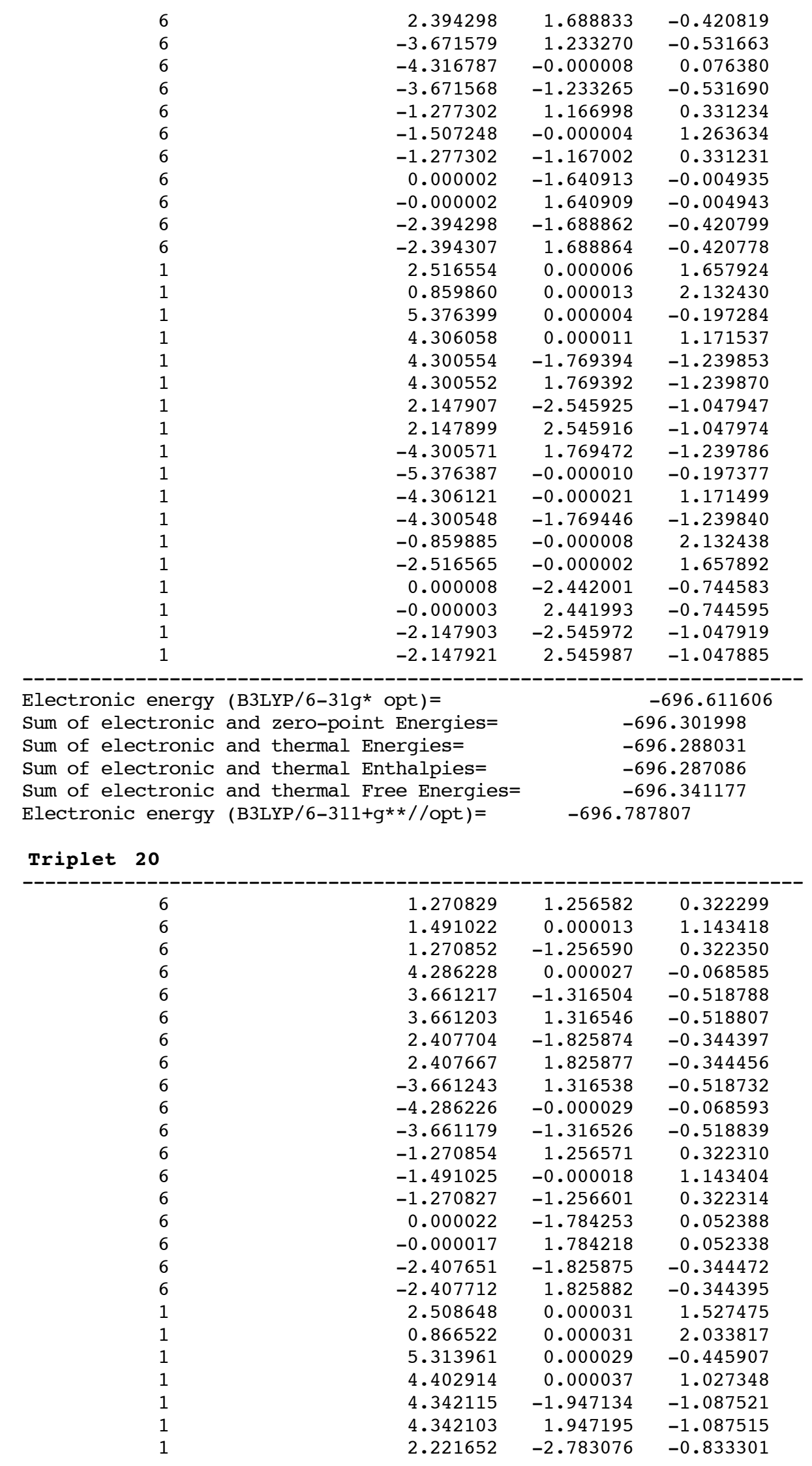




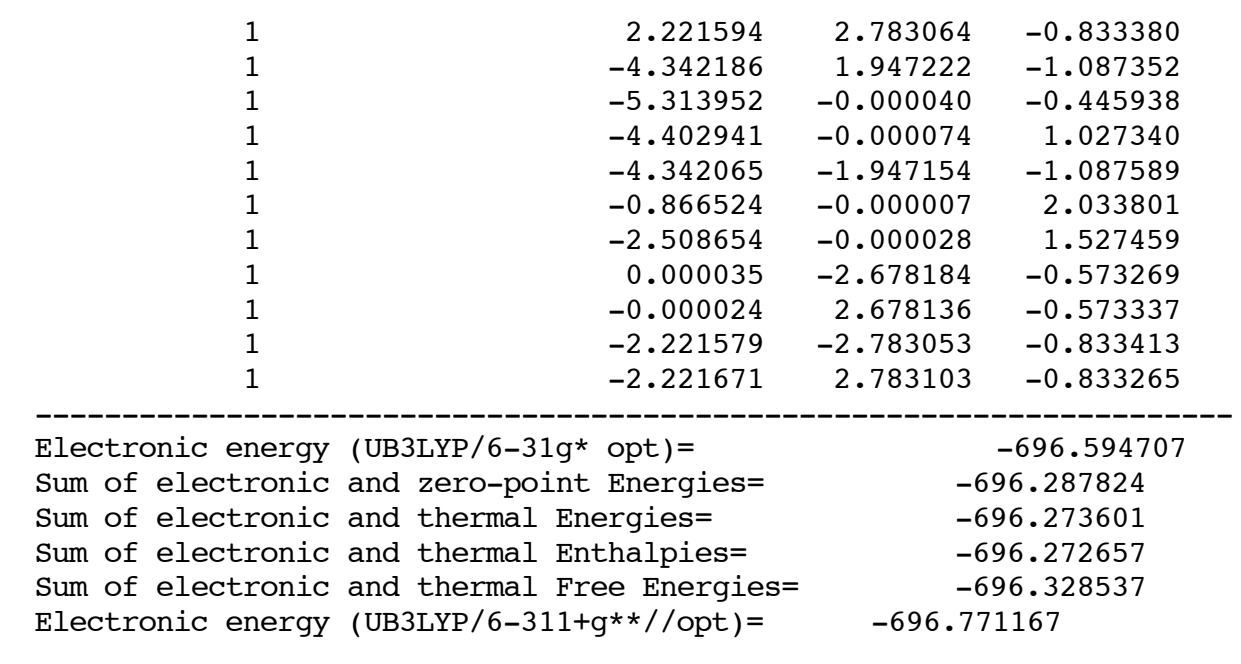

\section{Singlet 21}

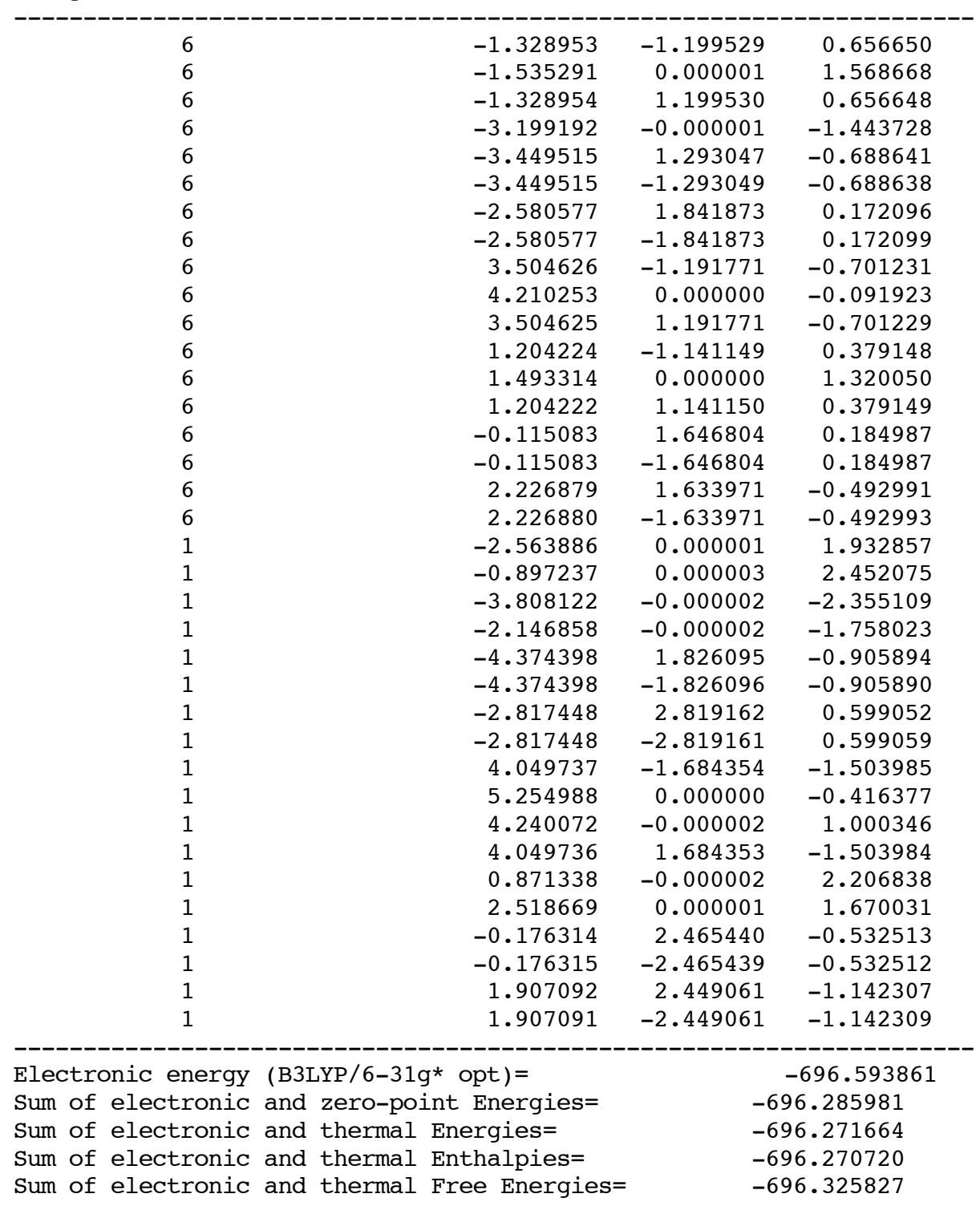


Electronic energy (B3LYP/6-311+g**//opt $)=\quad-696.770803$

Triplet 21

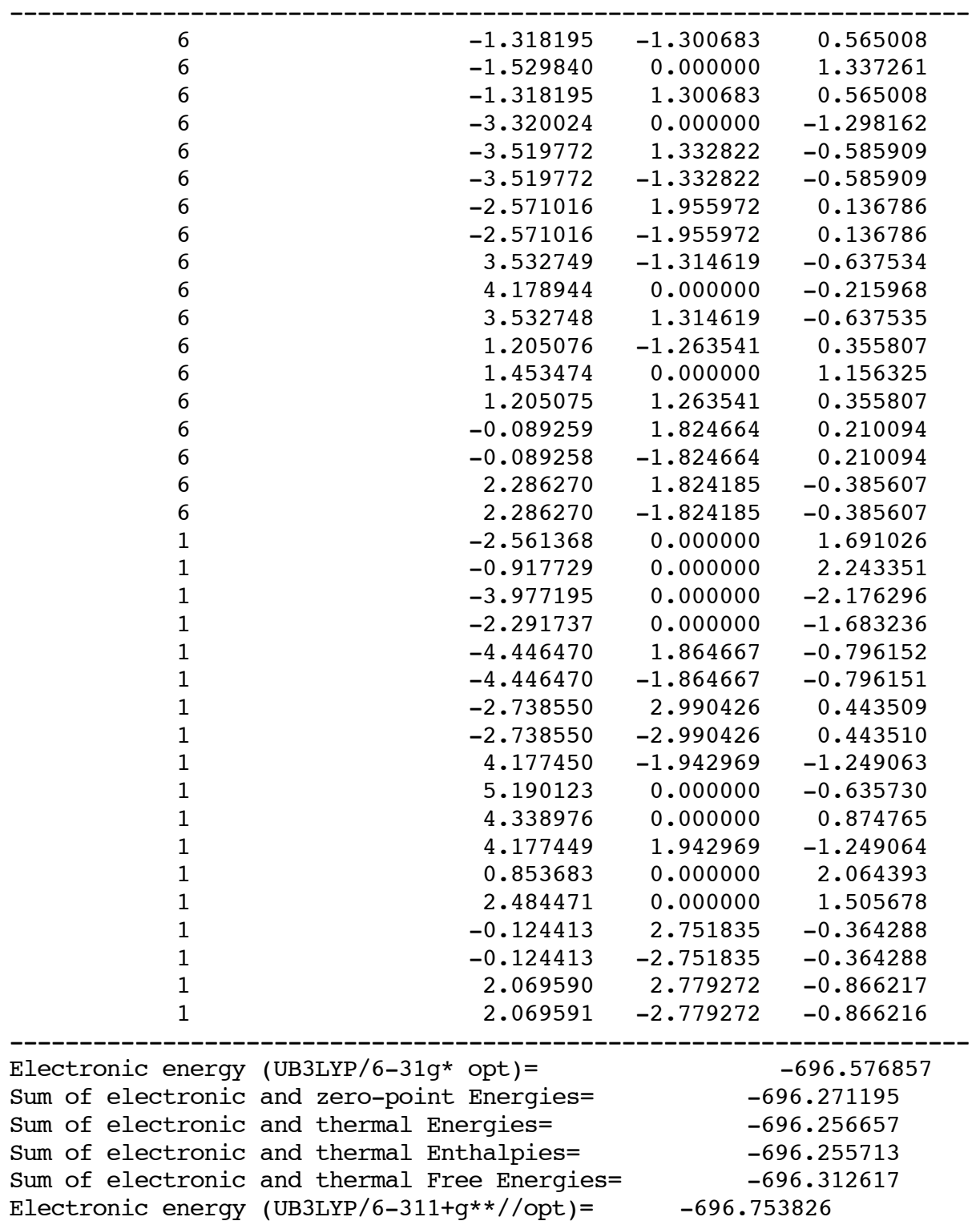

Singlet 22

$\begin{array}{rrrr}6 & -1.270717 & 1.126078 & -0.710817 \\ 6 & -1.541257 & 0.000055 & -1.678479 \\ 6 & -1.270763 & -1.126026 & -0.710868 \\ 6 & -2.925685 & -0.000020 & 1.633500 \\ 6 & -3.227526 & -1.281420 & 0.880909 \\ 6 & -3.227436 & 1.281468 & 0.881021 \\ 6 & -2.461099 & -1.776073 & -0.098775 \\ 6 & -2.460990 & 1.776151 & -0.098634 \\ 6 & 3.227544 & 1.281430 & 0.880877 \\ 6 & 2.925758 & 0.000021 & 1.633473 \\ 6 & 3.227458 & -1.281475 & 0.880989 \\ 6 & 1.270744 & 1.126010 & -0.710861 \\ 6 & 1.541245 & -0.000059 & -1.678480\end{array}$




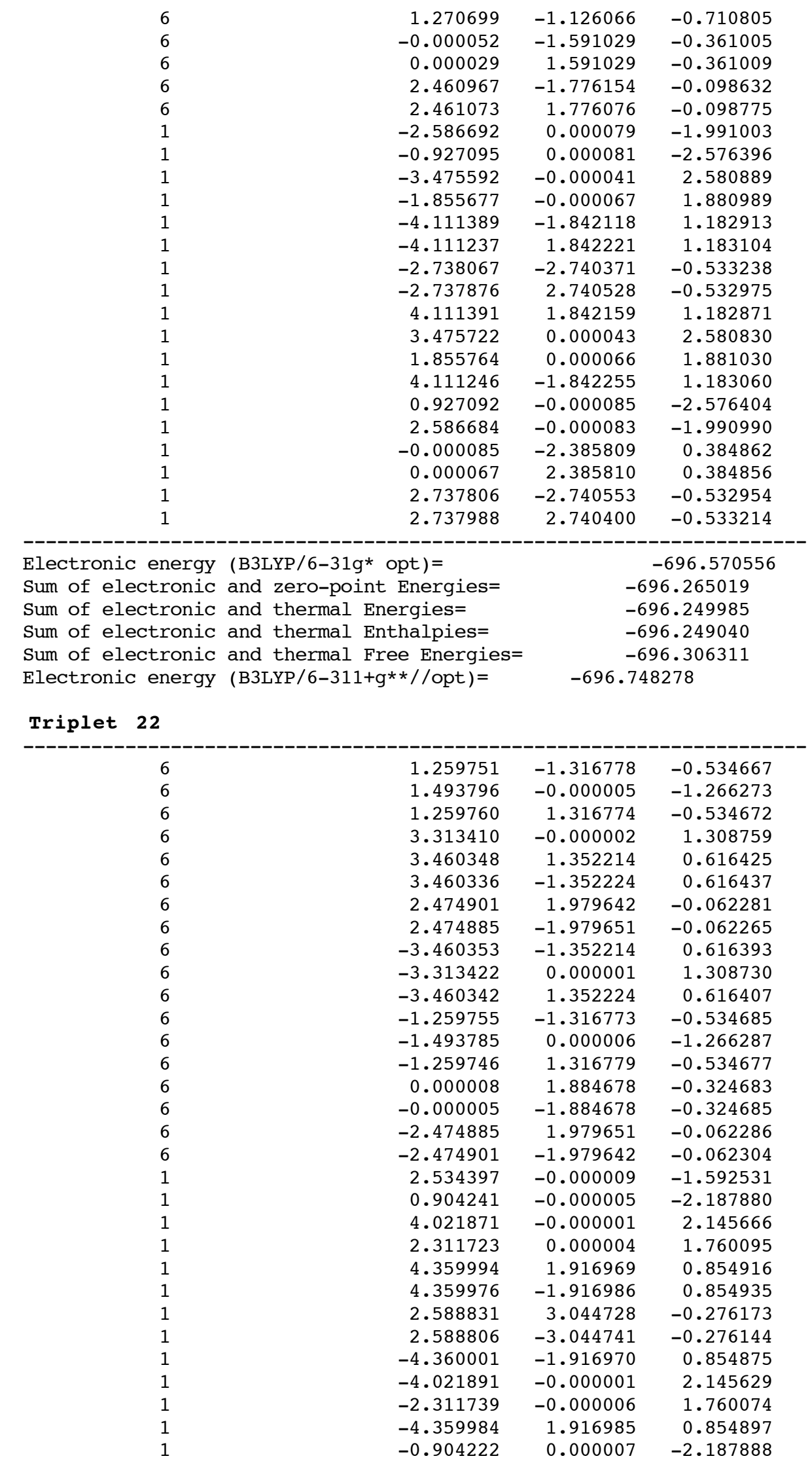




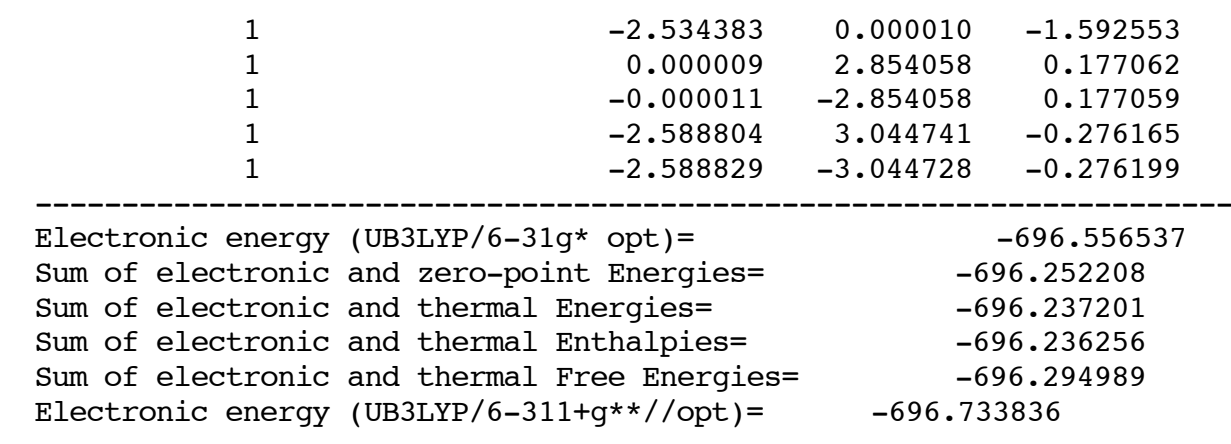

Singlet 23

\begin{tabular}{|c|c|c|c|}
\hline & 5.423934 & -0.000003 & -0.583460 \\
\hline & 4.679519 & -1.258877 & -1.005473 \\
\hline & 4.679528 & 1.258881 & -1.005461 \\
\hline & 3.467569 & -1.730902 & -0.618363 \\
\hline & 3.467577 & 1.730904 & -0.618355 \\
\hline & 2.511776 & -1.192815 & 0.330853 \\
\hline & 2.511778 & 1.192813 & 0.330853 \\
\hline & 2.933943 & -0.000001 & 1.163513 \\
\hline & -1.209131 & -1.675549 & 0.266472 \\
\hline & -1.209130 & 1.675546 & 0.266465 \\
\hline & 1.209130 & -1.675518 & 0.266455 \\
\hline & 1.209131 & 1.675514 & 0.266449 \\
\hline & 0.000017 & -1.183721 & 0.829321 \\
\hline & 0.000017 & 1.183720 & 0.829316 \\
\hline & -0.000007 & 0.000002 & 1.775839 \\
\hline & -3.467591 & -1.731007 & -0.618291 \\
\hline & -3.467594 & 1.731008 & -0.618290 \\
\hline & -2.511749 & -1.192850 & 0.330865 \\
\hline & -2.511749 & 1.192850 & 0.330861 \\
\hline & -2.933955 & 0.000001 & 1.163454 \\
\hline & -4.679509 & -1.258962 & -1.005431 \\
\hline & -4.679514 & 1.258966 & -1.005426 \\
\hline & -5.423894 & -0.000001 & -0.583595 \\
\hline & 6.390451 & -0.000004 & -1.097296 \\
\hline & 5.676279 & -0.000009 & 0.483904 \\
\hline & 5.167669 & -1.821824 & -1.798934 \\
\hline & 5.167687 & 1.821838 & -1.798909 \\
\hline & 3.120406 & -2.619700 & -1.145983 \\
\hline & 3.120419 & 2.619709 & -1.145968 \\
\hline & 4.007540 & -0.000002 & 1.313168 \\
\hline & 2.515684 & -0.000002 & 2.164008 \\
\hline & -1.067414 & -2.494755 & -0.438762 \\
\hline & -1.067413 & 2.494750 & -0.438772 \\
\hline & 1.067419 & -2.494695 & -0.438814 \\
\hline & 1.067422 & 2.494689 & -0.438823 \\
\hline & 0.854889 & 0.000004 & 2.437874 \\
\hline & -0.854966 & 0.000003 & 2.437790 \\
\hline & -3.120497 & -2.619911 & -1.145779 \\
\hline & -3.120502 & 2.619916 & -1.145775 \\
\hline & -2.515803 & 0.000002 & 2.163999 \\
\hline & -4.007570 & 0.000001 & 1.313008 \\
\hline & -5.167742 & -1.822039 & -1.798750 \\
\hline & -5.167753 & 1.822048 & -1.798737 \\
\hline & -5.676448 & -0.000003 & 0.483730 \\
\hline & -6.390324 & -0.000002 & -1.097596 \\
\hline $\begin{array}{l}\text { iic el } \\
\text { lect }\end{array}$ & $\begin{array}{l}\text { pt })= \\
\text { Energies= }\end{array}$ & \multicolumn{2}{|c|}{$\begin{array}{c}-889.525884 \\
-889.139652\end{array}$} \\
\hline
\end{tabular}


Sum of electronic and thermal Energies= Sum of electronic and thermal Enthalpies= Sum of electronic and thermal Free Energies= Electronic energy (B3LYP/6-311+g**//opt) =
$-889.122124$

$-889.121180$

$-889.182934$

$-889.747773$

Triplet 23

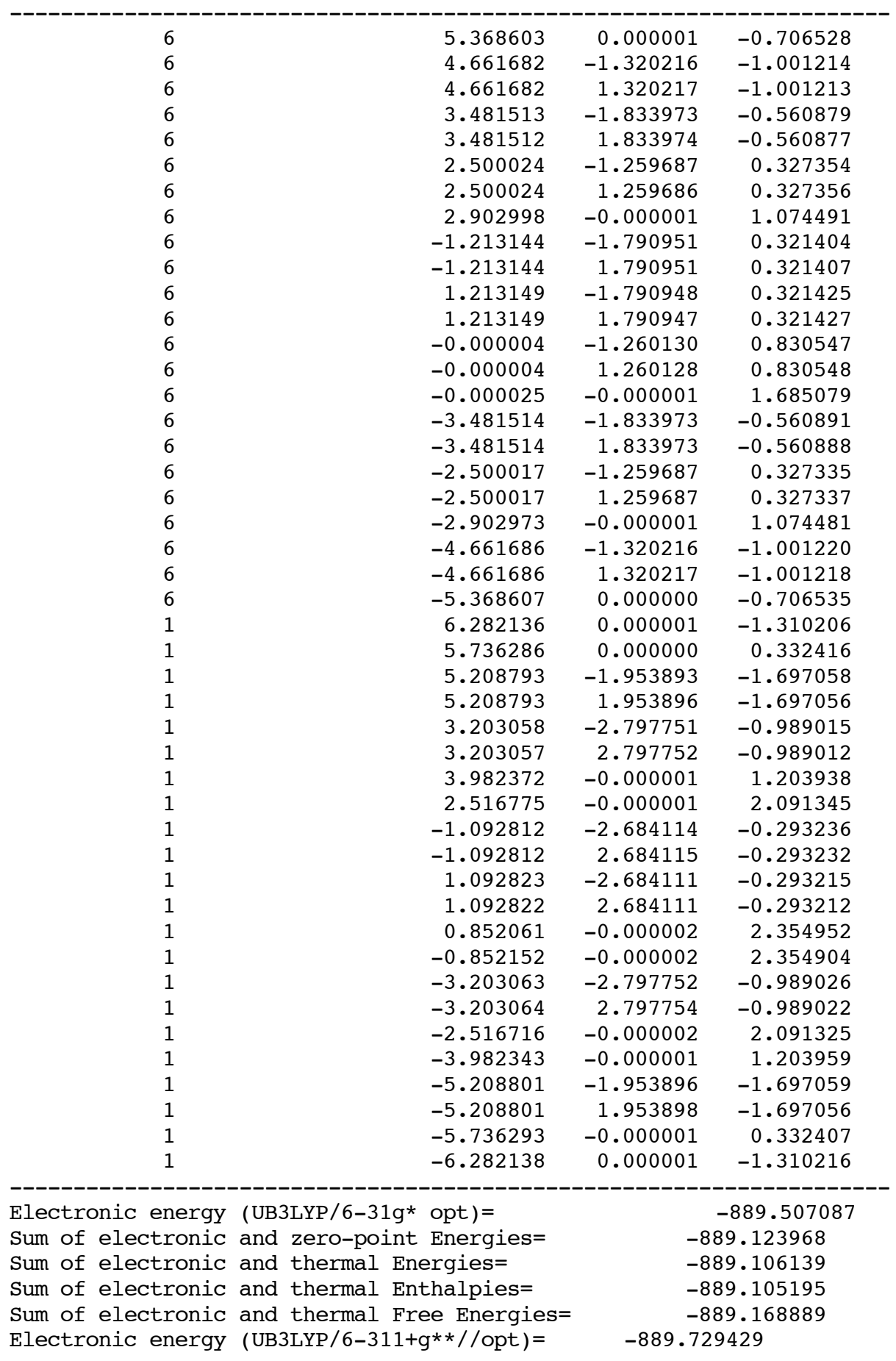




\section{Expanded Discussion of Singlet/Triplet and HOMO/LUMO Gaps.}

Although the singlet-triplet gaps (both computed and measured) for the polyacenes have been shown to correlate well with computed HOMO-LUMO gaps, ${ }^{\mathrm{S} 1}$ the singlet-triplet and HOMO-LUMO gaps computed for the $\square$-polyacenes do not correlate well. Like the situation for the normal polyacenes, the computed HOMOLUMO gaps for the four smallest $\square$-polyacenes $\left(7^{\prime}, n=0-3\right)$ do decrease (in both cases, the coefficients of the interacting polyene orbitals will decrease as the chains are lengthened, reducing the orbital splitting), but the computed singlet-triplet gaps for the $\square$-polyacenes are all comparable to each other (see Figure 1). We believe that this departure from normal polyacene behavior is due primarily to the increased flexibility of the frameworks that link the polyenes in the $\square$-polyacenes compared to those of the polyacenes. In the $\square$-polyacenes, the decreased inter-polyene orbital interactions upon lengthening allow the two polyene chains to move apart slightly and relieve some of the strain present in the linking framework. Note in Figure 1 how the interpolyene distances increase as the chains are lengthened, which also allows the $\mathrm{C}_{\text {polyene }}-\mathrm{C}_{\text {linker }}-\mathrm{C}_{\text {polyene }}$ angles to increase from $88^{\circ}$ in the barbaralane transition structure $\left(7^{\prime}, n=0\right)$ to $104^{\circ}$ in the tetramer $\left(7^{\prime}, n=3\right)$; the inter-polyene distances for the triplet oligomers change to a much smaller extent (and actually decrease slightly as the repulsive inter-polyene interactions present in the triplet states are relieved). The frameworks of the normal polyacenes are much more rigid, however. Thus, in the $\square-$ polyacenes, strain relief upon lengthening compensates for the decreased interpolyene orbital interaction, preventing the singlet-triplet gaps from decreasing as fast as the HOMO-LUMO gaps. In short, while the HOMO-LUMO gap is a property of a single structure (in this case, the ground state structure), two different structures (the optimized singlet and triplet) contribute to the singlet-triplet gap.

The HOMO and LUMO of the $\square$-polyacenes described are always orbitals of the type shown in Figure 2. The HOMO and LUMO of the normal polyacenes are not always the analogous inter-polyene combinations, however, because the polyenes are held closer together in the polyacenes, leading to a larger splitting away from the 
orbital frontier. The frontier orbitals are always still -like, however. The argument above is merely dependent on the fact that the inter-polyene distances in the normal polyacenes are comparable and do not change significantly as the chains are lengthened, while those in the $\square$-polyacenes change considerably and at different rates for the singlets and triplets.

(S1) (a) Wiberg, K. B. J. Org. Chem. 1997, 62, 5720-5727. (b) Houk, K. N.; Lee, P. S.; Nendel, M. J. Org. Chem. 2001, 66, 5517-5521 and references therein. 